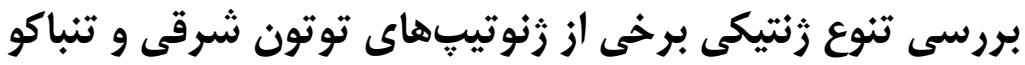

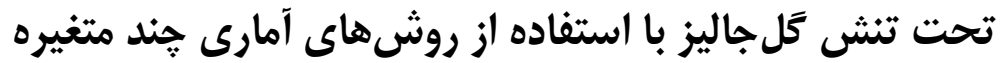

\author{
مريم طهماسب عالى '، امير فياض مقدمّ، رضا درويشز ادهّ و حسين عباسى هولاسوع
}

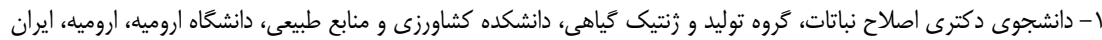

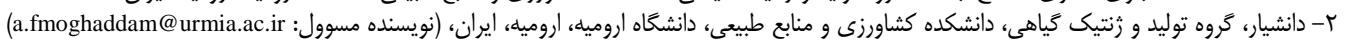

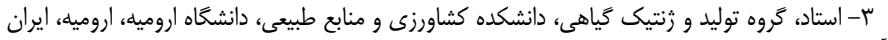

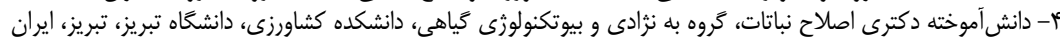

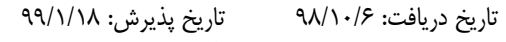

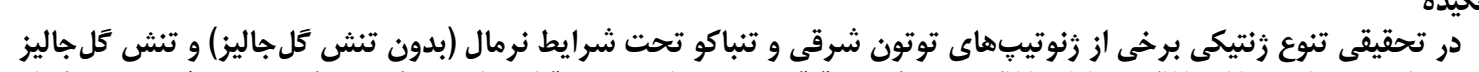

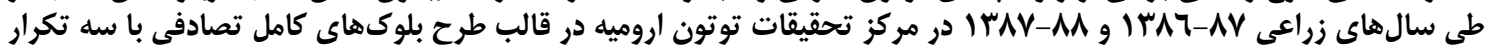

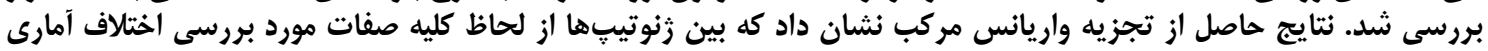

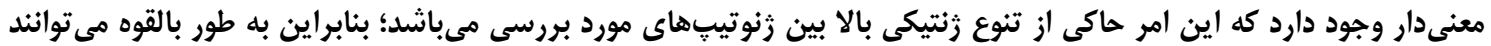

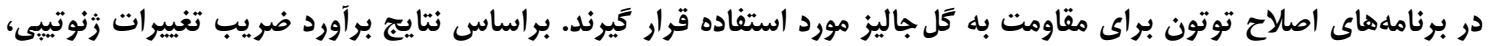

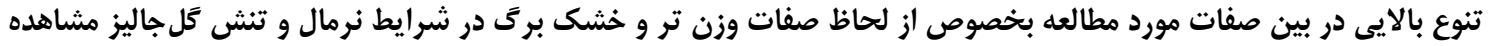

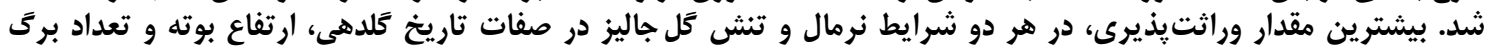

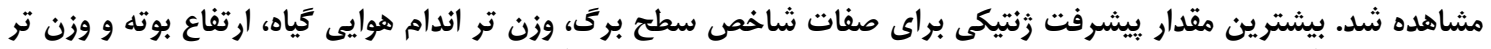

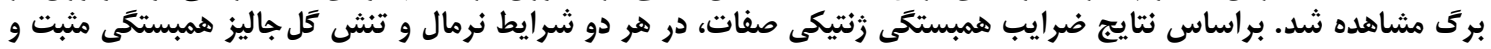

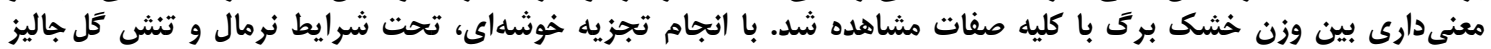

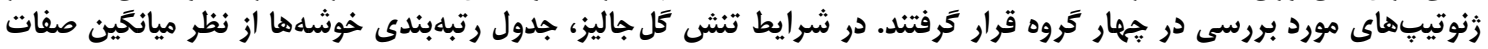

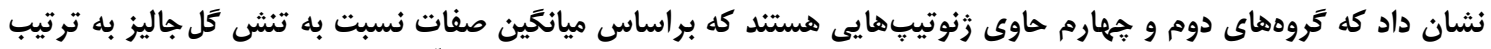

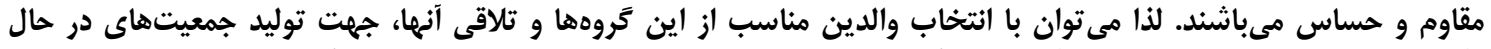

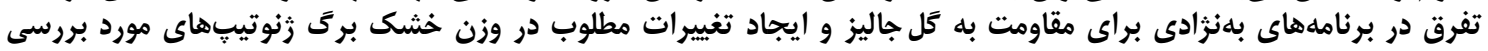

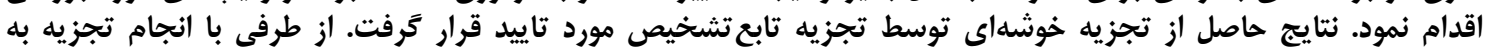

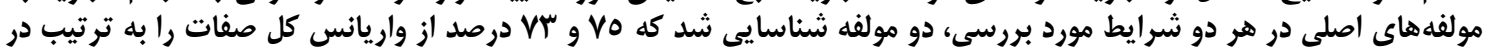

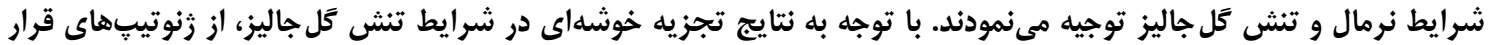

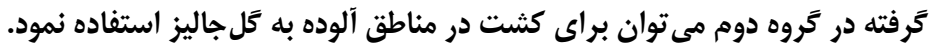

وازههاى كليدى: اصلاح براى مقاومت، يارازيت اجبارى، توتون، تنش زيستى، تجزيه حند متغيره، وراثتيذيرى

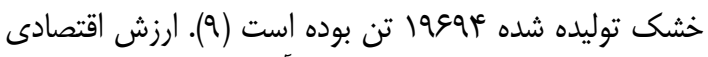

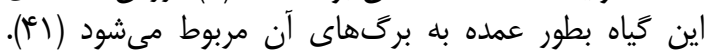

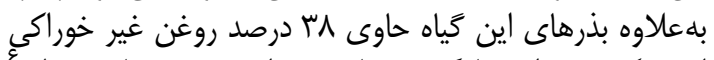

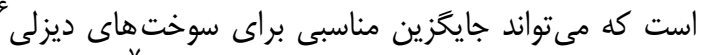

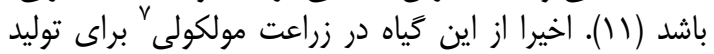

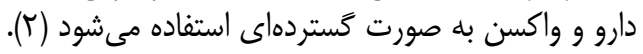

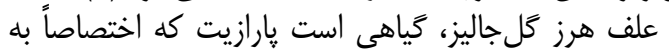

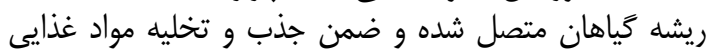

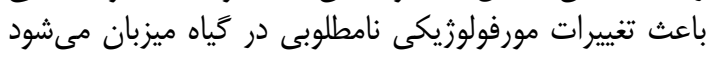

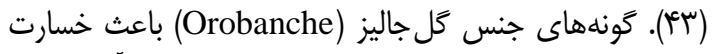

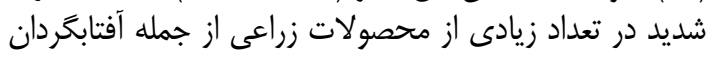
(Helianthus annuus L.) (Lycopersicon esculentum L.)

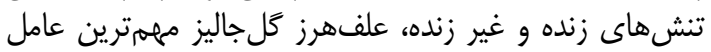

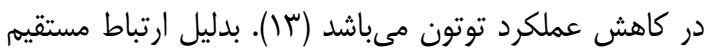

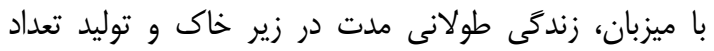

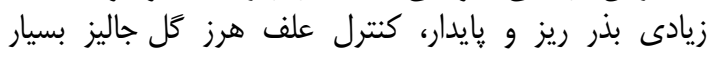
مشكل مىباشد. روشهاى مختلف از جمله زراعى، شئي ايميايى

1- Locus

5- Gene flow

2- Genetic variability 6- Biodiesel
مقدمه - مق

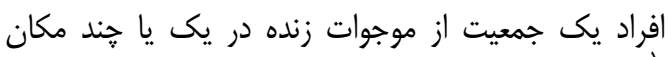

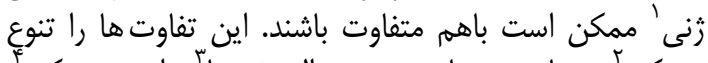

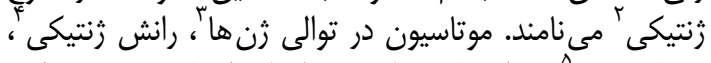

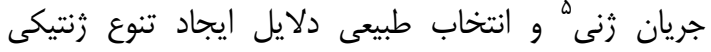

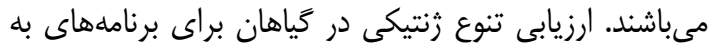

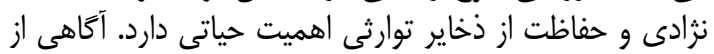

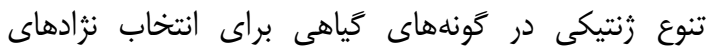

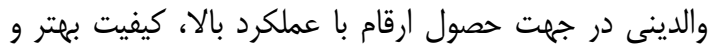

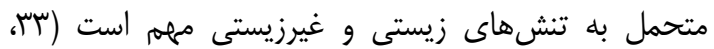
( (T/.TVG).

توتون (Nicotiana tabacum L.) يكى از مهرمترين

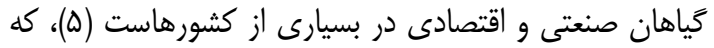

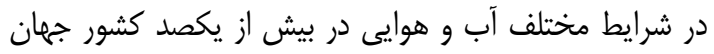

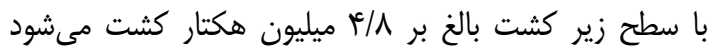

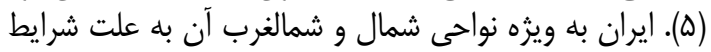

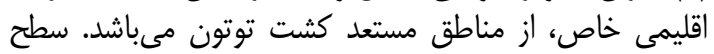

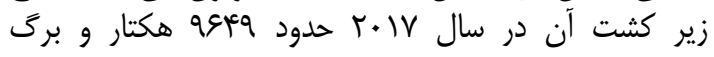

3- Genes sequence 4- Genetic drift 
غير از Vaba faba مكانيسم اصلى مقاومت در برابر كل جاليز

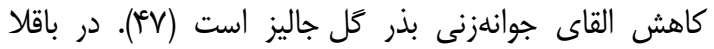

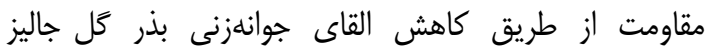

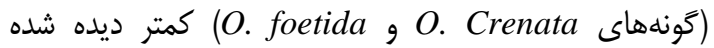
است. تا به امروز مكانيسمهاى مقاومت توصيف شده در باقلا، Pre-haustorial انگًل به بافتهاى ميزبان و قبل از توسعه هستوريوم (اركان

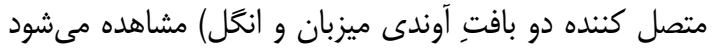

بررسى تنوع زنتيكى و تعيين روابط زنتيكى در مواد گياهى

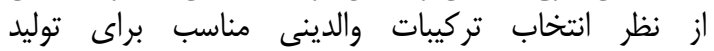

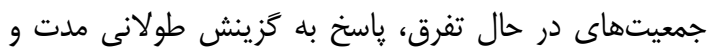

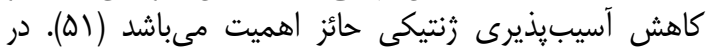

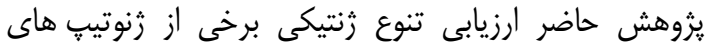

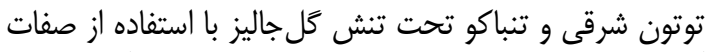

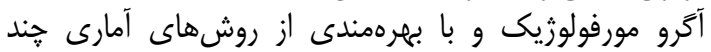
متغيره انجام گرفته است.

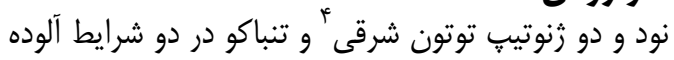

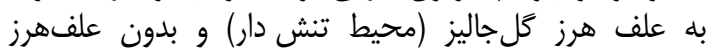

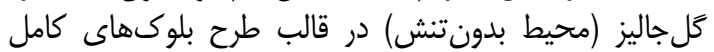

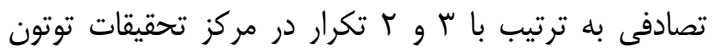

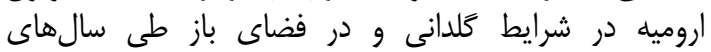
طول

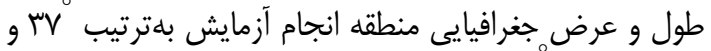

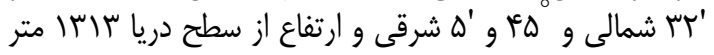

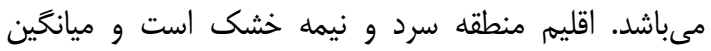

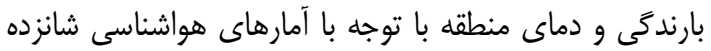

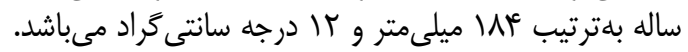

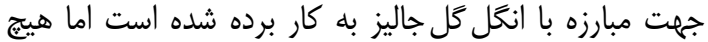

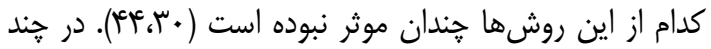

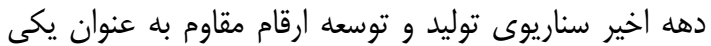

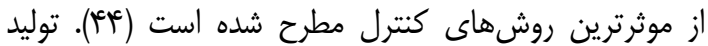

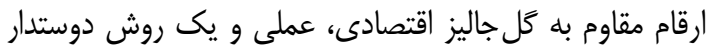

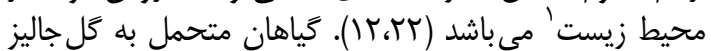

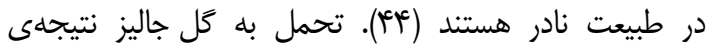

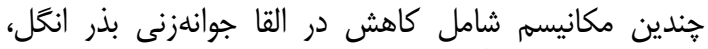

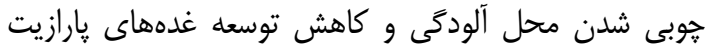
مىباشد (ع أF).

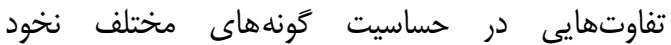
(Pisum spp.) (O. crenata Forsk) Crenate است (•†). در مطالعه اخير ززارش شده است كه مكانيسم

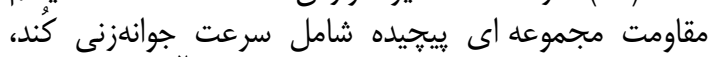

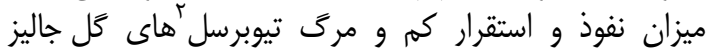
Crenate

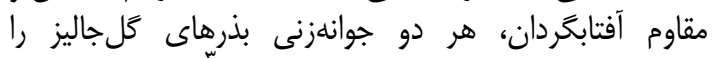

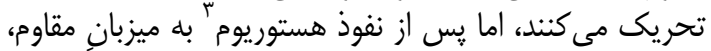

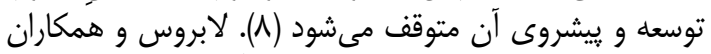

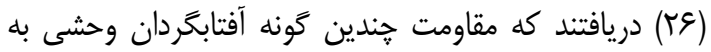

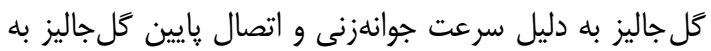

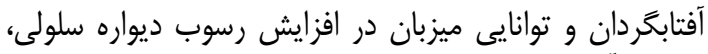

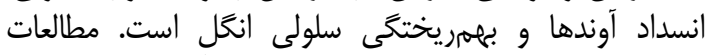

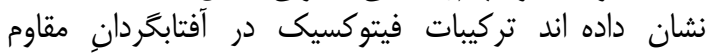

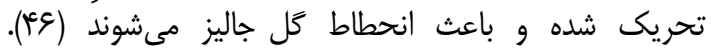

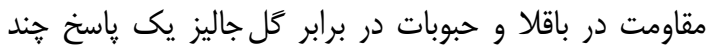

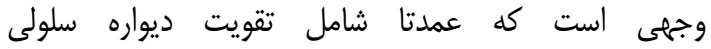

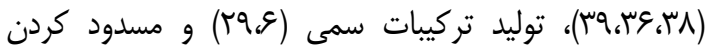

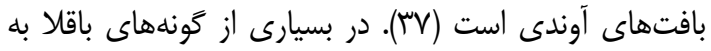


Table 1. The name of studied oriental and water pipe's tobacco genotypes

\begin{tabular}{|c|c|c|c|c|c|c|c|c|}
\hline كد & ز زنوتي״ & تيڤ & كد & رنوتيٍ & تيٍٍ & كد & رنوتيب & تيٍٍ \\
\hline 1 & Kharmanli 163 & شرقى & me & Samsun 959 & شرقى & $9 V$ & SPT 412 & شرقى (جُجِقُ) \\
\hline r & Nevrokop & شرقى & $r \Delta$ & Samsun dere & شرقى & $9 \wedge$ & Esfahan5 & تنباكو \\
\hline r & Trabozan & شرقى & 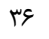 & OR-205 & شرقى & 99 & SPT 420 & شرقى (جُيُق) \\
\hline f & Krumovgraid & شرقى & rv & OR-345 & شرقى & $\checkmark \cdot$ & SPT 430 & 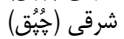 \\
\hline$\Delta$ & Basma.S.31 & شرقى & щ & OR-379 & شرقى & $n$ & SPT 432 & شرقى (پُِيقن) \\
\hline 9 & Triumph & شرقى & rq & C.Н.T.209.12e & شرقى & Vr & SPT 433 & شرقى (جِيُقُ) \\
\hline$\checkmark$ & Xanthi & شرقى & c. & C.H.T.209.12exF.K.40-1 & شرقى & $v^{m}$ & SPT 434 & شرقى (جِيُق) \\
\hline$\wedge$ & Matianus & شرقى & (i) & C.H.T.266-6 & شرقى & $v^{e}$ & SPT 436 & شرقى (جُجِقُ) \\
\hline 9 & Immni 3000 & شرقى & et & С.Н.T.283-8 & شرقى & $\checkmark \Delta$ & SPT 439 & شرقى (هُيُق) \\
\hline 1. & Melkin 261 & شرقى & سع & C.Н.T.273-38 & شرقى & Ve & SPT 441 & شرقى (جِيُق) \\
\hline 11 & Tyk-Kula & شرقى & pe & Basma 12-2 & شرقى & V & Esfahan2 & تنباكو \\
\hline ir & Ss-289-2 & شرقى & $i \Delta$ & Basma 16-10 & شرقى & v^ & SPT 413 & شرقى (جُجِقُ) \\
\hline سו & Ohdaruma & شرقى & is & Basma 104-1 & شرقى & vq & Esfahani & تنباكو \\
\hline $1 f$ & Ploudive 58 & شرقى & er & Basma 181-8 & شرقى & $\wedge$. & Jahrom14 & تنباكو \\
\hline 10 & Line 20 & شرقى & $\uparrow \wedge$ & Zichna & شرقى & $\wedge 1$ & Borazjan & تنباكو \\
\hline 19 & T-B-22 & شرقى & pq & Izmir & شرقى & NT & L $16 b$ & شرقى \\
\hline IV & Ts 8 & شرقى & $\Delta$. & P.D. 324 & شرقى & 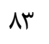 & Balouch & تنباكو \\
\hline$M$ & Alborz23 & شرقى & 01 & P.D. 325 & شرقى & NF & Lengeh & تنباكو \\
\hline 19 & F.K. $40-1$ & شرقى & Qr & P.D.406 & شرقى & $\Lambda \Delta$ & Saderati & تنباكو \\
\hline r. & Pz17 & شرقى & Wr & P.D. 328 & شرقى & $\Lambda \varepsilon$ & Eraghi & تنباكو \\
\hline rI & K.P.На & شرقى & DF & P.D.329 & شرقى & AV & Shahroudi & تنباكو \\
\hline tr & K.B & شرقى & $\Delta \Delta$ & P.D.336 & شرقى & $M$ & T.K.L & شرقى \\
\hline זr & G.D. 165 & شرقى & $\Delta \varphi$ & P.D. 345 & شرقى & 19 & L 17 & شرقى \\
\hline rF & H.T.I & شرقى & $\Delta V$ & P.D.364 & شرقى & $q$. & С.Н.Т.269-12e & 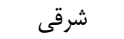 \\
\hline ra & Kramograd N.H.H. 659 & شرقى & $\Delta \Lambda$ & P.D.365 & شرقى & 91 & Samsun 1 & شرقى \\
\hline r & T.K.23 & شرقى & $\Delta q$ & P.D. 371 & شرقى & q4 & Samsun Katenizi & شرقى \\
\hline tr & L 16a & شرقى & c. & P.D.381 & شرقى & & & \\
\hline rA & Izmir 7 & شرقى & 9) & SPT 403 & شرقى (خُشْقِ) & & & \\
\hline rq & Mutant 3 & شرقى & gr & SPT 405 & شرقى (جُشْقِ) & & & \\
\hline r. & Mutant 4 & شرقى & s & SPT 406 & شرقى (خُجِقُ) & & & \\
\hline ו & Pobeda 1 & شرقى & st & SPT 408 & شرقى (جُشيقق) & & & \\
\hline זr & Pobeda 2 & شرقى & 90 & SPT 409 & شرقى (جُشْقِ) & & & \\
\hline 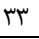 & Rustica & تنباكو & 99 & SPT 410 & شرقى (جُشيُق) & & & \\
\hline
\end{tabular}

شدند. پِ از آزمون توزيع نرمال اشتباهات و همكنى واريانس

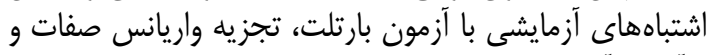

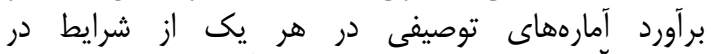

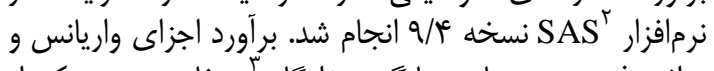

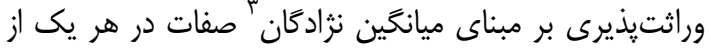

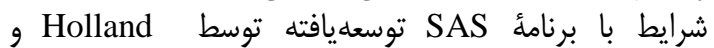
همكاران (1) انجام كرفت. برنامه توسعه يافته درفت در سايت بال لينك

http://www4.ncsu.edu/ jholland/heritability/Inbre

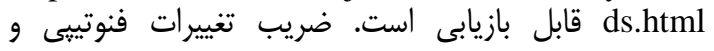

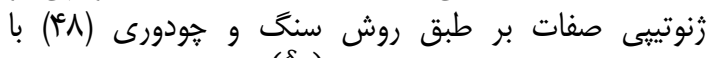
استفاده از فرمولهاى

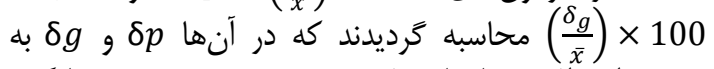

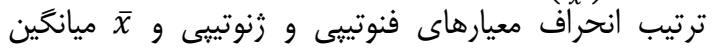

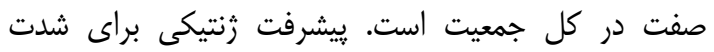

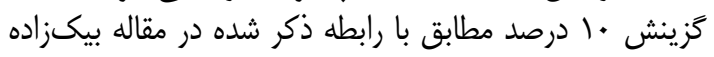
و همكاران ( () با استفاده از رابطه زير محاسبه شد شد. $\mathrm{GC}=\mathrm{K} \times \mathrm{h}_{\mathrm{b}}^{2} \times \frac{\sqrt{\delta_{\mathrm{p}}^{2}}}{\overline{\mathrm{x}}} \times 100$
براى انجام آزمايش در هر يك از سالها و در هر يك از

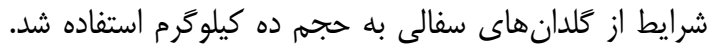

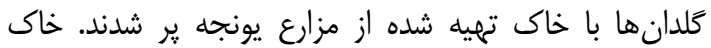

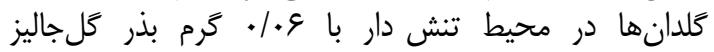
مخلوط شد. نشاء هر يك ازئ (Orobanche cernua)

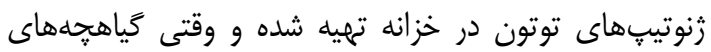

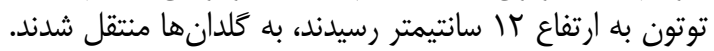

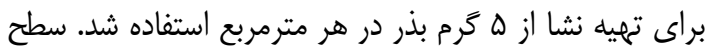
خزانه بعد از بذر ياشى با بر يكى لايه كود كوسفندى يوسيده يوشيده شد.

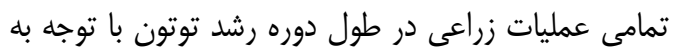

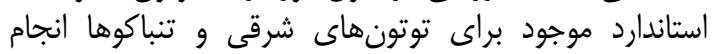

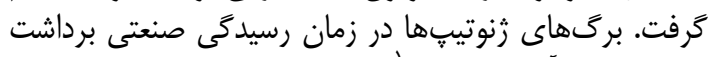

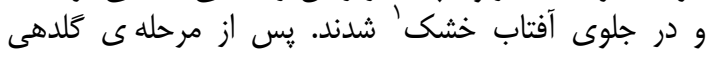

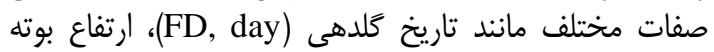

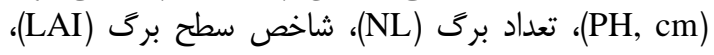

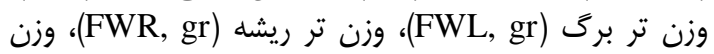

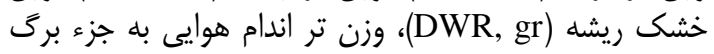
(APFW, gr)

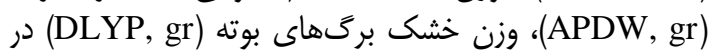

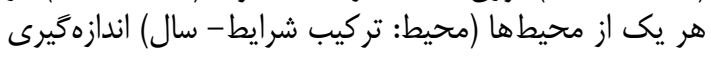


و تعداد برگ در بوته داراى بيشترين ضريب تغييرات فنوتييى

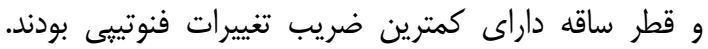
وجود تنوع بالا در صفات مختلف توسط حسينزاده داد فشالمى و

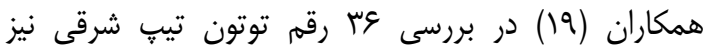

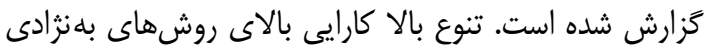

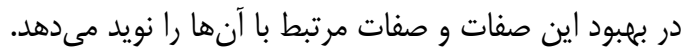

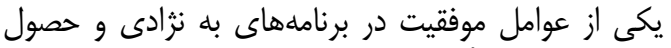

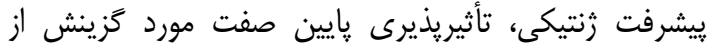

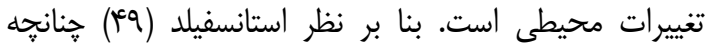

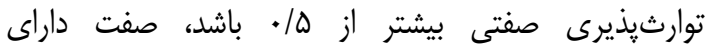

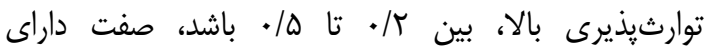

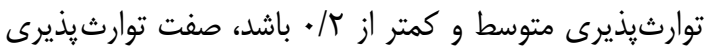

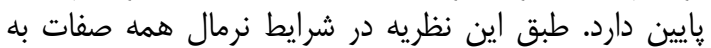

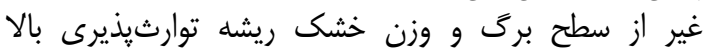

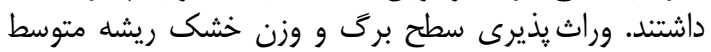

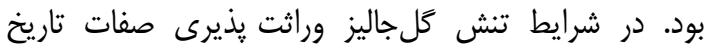

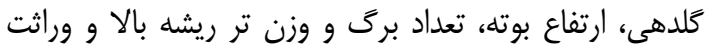
يذيرى سطح برى، وزن خشكى ريش ريشه، وزن تر تر برى

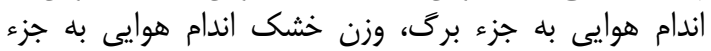

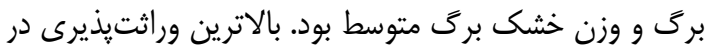

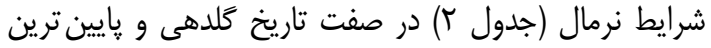

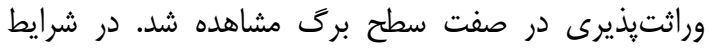

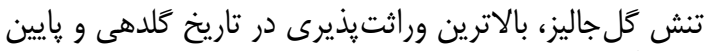

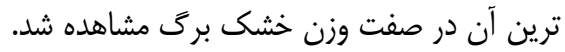

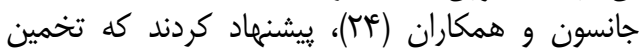

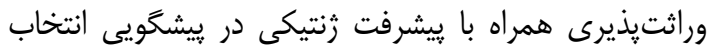

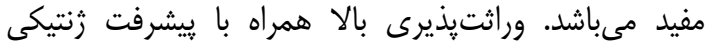

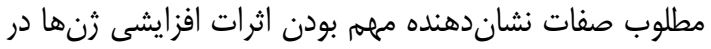

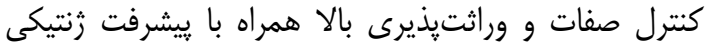

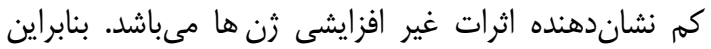

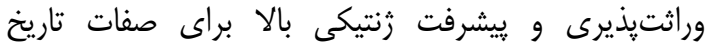

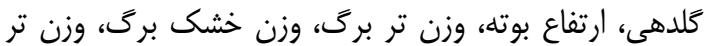

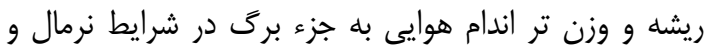

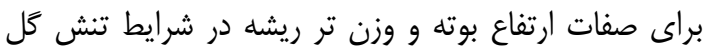

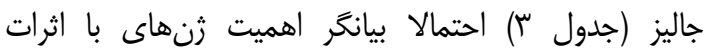

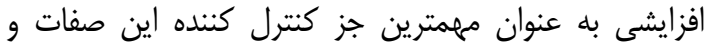

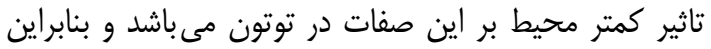

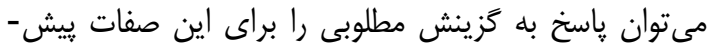

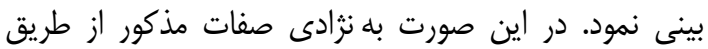

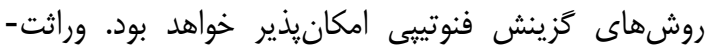

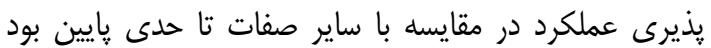

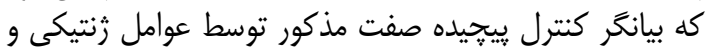

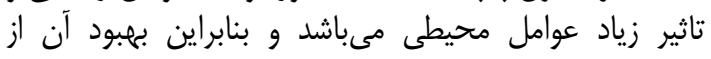

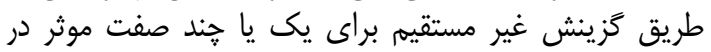

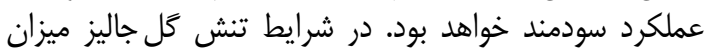

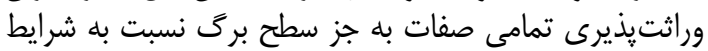

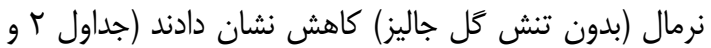

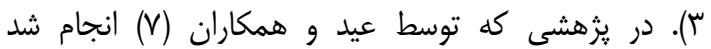
كزارش كردند برخى صفات كه در شرايط نرمال وراثتيذيرى تونى

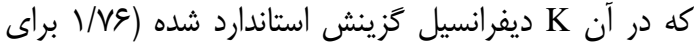
. واريانس فنوتييى و

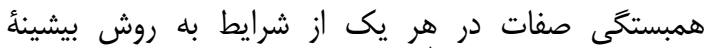

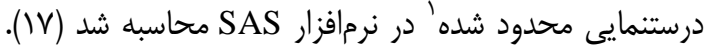

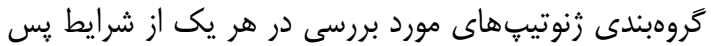

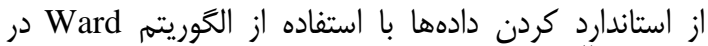

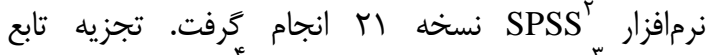

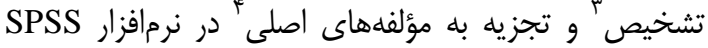

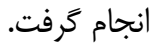

\section{نتايج و بحث} تجزيه واريانس و آمارههاي توصيفى صفات

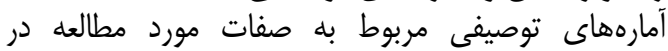

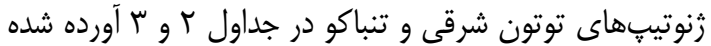

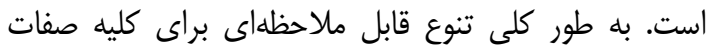

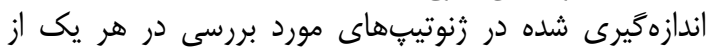

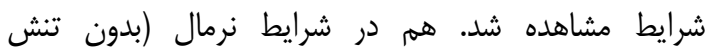

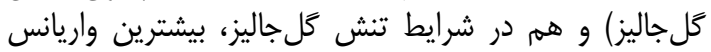

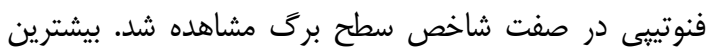

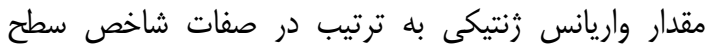

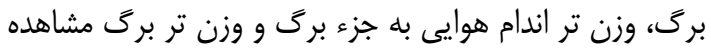

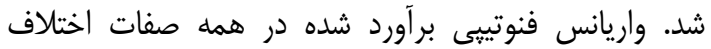

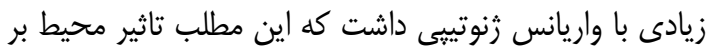

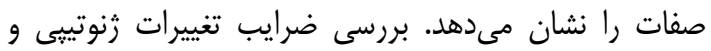

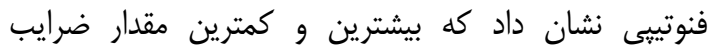

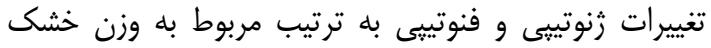

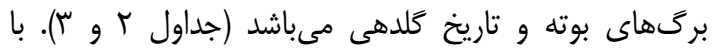

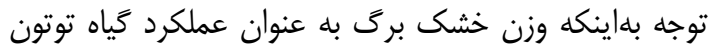
محسوب مىشود و ساير صفات مورفولوزيكى بن با آن آن رابطه

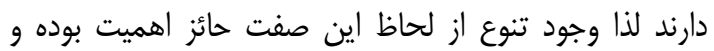

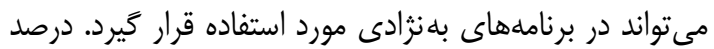

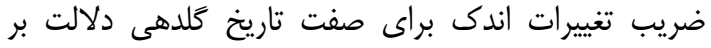

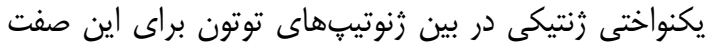

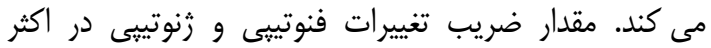

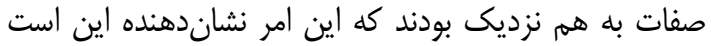

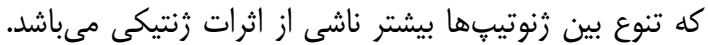

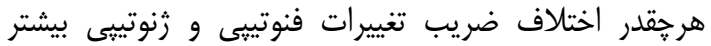

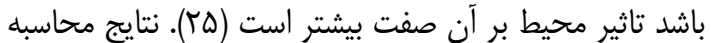

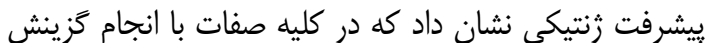

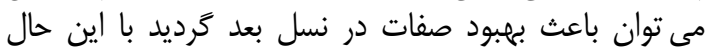

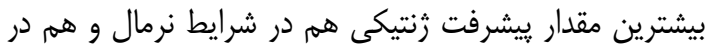

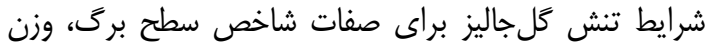

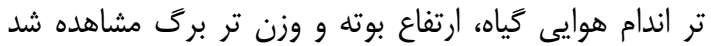

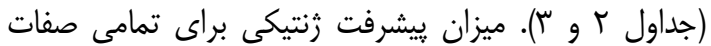

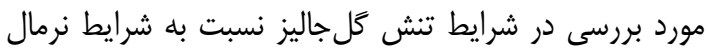

كمتر بود.

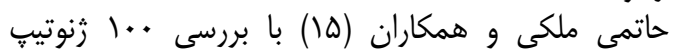

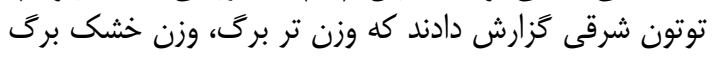


تجزيه واريانس در شرايط نرمال (بدون تنش كل جاليز) و وإي

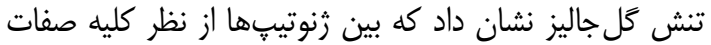

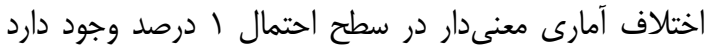

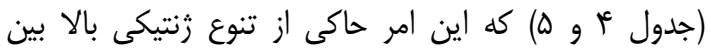

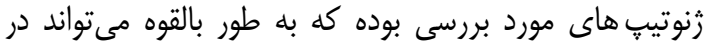
برنامههاى بلهنزادى براى مقاومت به بلى بل جاليز مورد استفاده

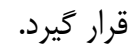

و بازده زنتيكى مطلوبى داشتند در شرايط تنش ميزان بازده إني

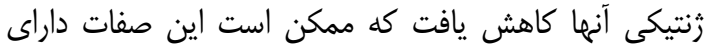

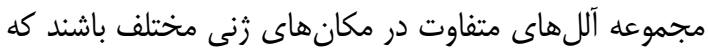

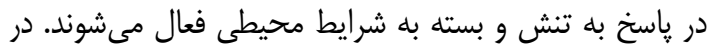

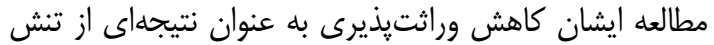

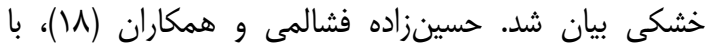

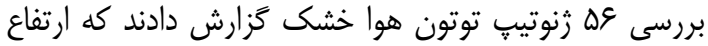

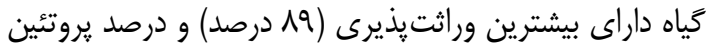

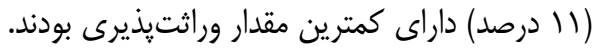

جدول r- آمارههاى توصيفى صفات مورد مطالعه در زنوتيڤهاى توتون شرقى و تنباكو تحت شرايط نرمال (بدون تنش كلجاليز) Table 2. Descriptive statistics for studied traits in oriental and water pipe's tobacco genotypes under normal (without Orobanche stress) conditions

\begin{tabular}{|c|c|c|c|c|c|c|c|c|}
\hline \multirow{2}{*}{ يِيشرفت زنتيكى } & \multicolumn{2}{|c|}{ ضريب تغييرات (\%) } & \multirow{2}{*}{ وراثتيذيرى عمومى } & \multicolumn{3}{|c|}{ واريانس } & \multirow{2}{*}{ ميانغين } & \multirow{2}{*}{ صفات } \\
\hline & زنوتييى & فنوتييى & & محيطى & ز زنوتيبى & فنوتييى & & \\
\hline $11 \% \cdot 9$ & $11 / 99$ & Ir/Ag & $\cdot / \mathrm{M}$ & r/^9 & sr/VI & $V / 4$ & $9 \cdot / 91$ & FD \\
\hline מז/r & $r N / F \Lambda$ & $T M / D F$ & . /AT & $1 \varepsilon / 4 q$ & 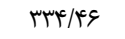 & سז/. & gF/Tr & $\mathrm{PH}$ \\
\hline$৭ / \wedge \Delta$ & $r q / r \Delta$ & $M r / \Lambda$. & $\cdot|v|$ & $r F / \& \Lambda$ & $+r / q$. & ST/Ir & Tr/GD & NL \\
\hline $1 \cdot r T / \cdot V$ & $r V / \cdot r$ & $9 q / 4$. &.$/ 18$ & $1 \cdot f|f| \cdot / v q$ & $V T \mid \Delta G \cdot / V$. & IDQTVEF $/ \cdot 1$ & $119 / 11$ & LAI \\
\hline$r \Delta / \& q$ & $\Delta s / V q$ & $V T / \cdot \Delta$ & . /gr & $V \& \Psi / . q$ & $1 \cdot v 9 / v$ & IVru & $\Delta V / \Lambda S$ & FWL \\
\hline $11 / v r$ & $q \cdot / 4 q$ & VN/DT & .109 & $r q / v \Delta$ & $V Q / \& \Lambda$ & ITV/OT & $\mid f / \mu \wedge$ & DLYP \\
\hline $10 / V \&$ & $r V / \Delta$. & $r \& / V \Delta$ & . /9F & F & ITA/QT & $190 / 94$ & rq/qr & FWR \\
\hline$r / q 1$ & 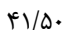 & $\varepsilon \cdot / 4$ &.$/ 4 r$ & $|N| \cdot r$ & $|9| 9 \mid$ & $r \Delta / \Lambda$ & q/Ar & DWR \\
\hline$r \cdot / v g$ & Frefre & $\Delta V / T$ & $\cdot / \Delta V$ & $q \cdot v / v r$ & $q 44 / r q$ & $190 \cdot / 9$. & $v \cdot / q$. & APFW \\
\hline $9 / V r$ & FI/.r & $\Delta F / . q$ & $\cdot / \Delta \Lambda$ & $p \Delta / \& q$ & $\Delta T / I F$ & $q \cdot / q 4$ & $\mid \mathrm{V} / \mathrm{s}$. & APDW \\
\hline
\end{tabular}

جدول ب- آمارههاى توصيفى صفات مورد مطالعه در زنوتيبٍ هاى توتون شرقى و تنباكو تحت تنش كل جاليز Table 3. Descriptive statistics for studied traits in oriental and water pipe's tobacco genotypes under Orobanche stress conditions

\begin{tabular}{|c|c|c|c|c|c|c|c|c|}
\hline \multirow{2}{*}{ ييشرفت زنتيكى } & \multicolumn{2}{|c|}{ ضريب تغييرات (\%) } & \multirow{2}{*}{ وراثتيذيرى عمومى } & \multicolumn{3}{|c|}{ واريانس } & \multirow{2}{*}{ ميانگين } & \multirow{2}{*}{ صفات } \\
\hline & زنوتيبى & فنوتيبى & & محيطى & زنوتييى & فنوتييى & & \\
\hline 9/9 & $1 \cdot / v 8$ & $11 / 90$ & $\cdot|\lambda|$ & G/א & rq/rq & FN/DT & $\Delta \Lambda / T \Lambda$ & FD \\
\hline$r \cdot \mu$ & سوו & rq/qu & س & ITr/OT & trI/.r & $\Gamma \Delta \Psi / \cdot 1$ & pr & $\mathrm{PH}$ \\
\hline N/AT & rN/AT & $m y / q g$ & $\cdot \mid \& \Lambda$ & $|9 / r|$ & re/qF & $\Delta F / r V$ & $r / / .9$ & NL \\
\hline $11 \cdot 1 \Delta 9$ & ه & rN/Tq & $\cdot / 4 \wedge$ & $\Delta S \mid G \wedge Y / \Delta$. & $p r q . \wedge r / 1$. & $q r \cdot \Delta V V$ & $1918 / V T$ & LAI \\
\hline $19 / 19$ & Tr/m & $\Delta Q / T r$ & $\cdot / \mu$. & $F \Delta \varepsilon / q V$ & $r \Lambda F / \Delta V$ & $q 4 \cdot / r V$ & $\Delta 1 / 9 q$ & FWL \\
\hline F/AT & pe/me & 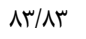 & $\cdot / T \Lambda$ & (q/Vq & re/V & $Q \Delta / \Delta T^{c}$ & $11 / 99$ & DLYP \\
\hline $11 / \mu$. & צץ & TH/N & $\cdot / \Delta \Delta$ & s广/r & $V Q / g Y^{c}$ & هـ/عسו & $r e /$ Le & FWR \\
\hline$r / \Lambda$. & T & GT/Mr & e & $\mid f / g f^{f}$ & V/rg & rI/Ne & $V / \Delta$. & DWR \\
\hline TH/DI & $r N / V^{\mu}$ & $F T / 90$ &.$/ 10$ & एq)/^. & rqז/rA & $M I / I V$ & $9 q / 1 r$ & APFW \\
\hline$\Delta / \& \&$ & $r \cdot / 1 r$ & "r &.$/ \pi V$ & إعا. & $r \Delta / Q \Lambda$ & $V \cdot / r T$ & $I T / V \cdot$ & APDW \\
\hline
\end{tabular}

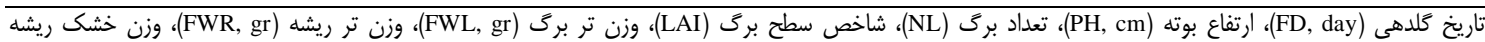

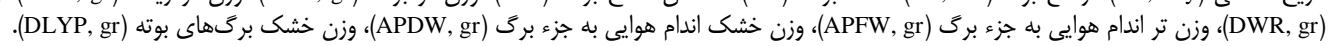

جدول ع - ميانگين مربعات صفات مورد بررسى در زنوتيبهاى توتون شرقى و تنباكو تحت شرايط نرمال (بدون تنش گل جاليز) Table 4. Mean squares of studied traits in oriental and water pipe's tobacco genotypes under normal (without Orobanche stress) conditions

\begin{tabular}{|c|c|c|c|c|c|c|c|c|c|c|c|}
\hline \multicolumn{10}{|c|}{ ميانخين مربعات } & \multirow{2}{*}{ درجه آزادى } & \multirow{2}{*}{ منابع تغييرات } \\
\hline APDW & APFW & DWR & FWR & DLYP & FWL & LAI & NL & $\mathrm{PH}$ & FD & & \\
\hline $91 / .9$ & $\mid r V / r F^{2}$ & $1 \cdot \Delta / \cdot r^{*}$ & $\Delta r H / Q I^{*}$ & $\Delta q \cdot / 4 \Lambda^{*{ }^{*}}$ & $|0 \cdot / \mu|$ & FeFTr/T. & $1 \cdot / f \Delta$ & $V T / F Q$ & $\mathrm{rV} / \mathrm{IV}^{*}$ & 1 & سال \\
\hline 4.119 & $10 \cdot / T V$ & If/re & FN/TA & $\mathrm{N} / \mathrm{VV}$ & $M M I / I F$ & pForq./q. & F/Tg & $r q / \Delta q$ & س & r & | اشتباه | \\
\hline$T Y V / T \varphi^{* * * 3}$ & TTAT/GY"* & $\Lambda \varepsilon / \Delta \Gamma^{* * *:}$ & $\triangle \Delta Q / V V^{* * *}$ & & YAVT/KY"*\% & HETI\&G./D."** & IAV/T & $\mid f \cdot r / \cdot 9^{* \% *}$ & TEQ/GY" & 91 & زنوتيب \\
\hline Tr/DT & $\Delta \cdot \Delta / \& \Lambda$ & $19 / 48$ & $\Delta F / 1$. & 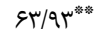 & $\Delta Q M / \Delta T$ & GATEY./T. & $I T / \cdot V$ & $G \Delta / T_{\Delta}$ & $\mid r / F \Lambda^{*}$ & १) & رنوتيّ × سال \\
\hline$\Delta \Delta / T r$ & $q \cdot v / v r$ & IN/V^ & NH/re & rq/va & $V G \Psi / \cdot 9$ & $1 . r \varepsilon \cdot F \psi / \mu$. & MT/gY & $1 \varepsilon / F q$ & F/AV & MT & اشتباه r \\
\hline
\end{tabular}

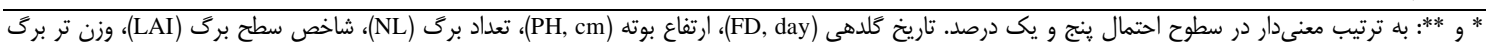

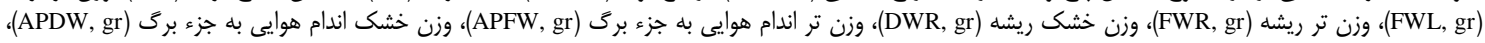
وزن خشك برگهاى بوته (DLYP, gr). 
جدول ه- ميانكَين مربعات صفات مورد بررسى در زنوتيبهاى توتون شرقى و تنباكو تحت تنش كل جاليز Table 5. Mean squares of studied traits in oriental and water pipe's tobacco genotypes under Orobanche stress conditions

\begin{tabular}{|c|c|c|c|c|c|c|c|c|c|c|c|}
\hline \multicolumn{10}{|c|}{ ميانگين مربعات } & \multirow{2}{*}{ درجه آزادى } & \multirow{2}{*}{ منابع تغييرات } \\
\hline APDW & APFW & DWR & FWR & DLYP & FWL & LAI & NL & $\mathrm{PH}$ & FD & & \\
\hline$V \wedge V / T \Lambda^{* * * *}$ & 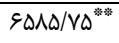 & $119 / V^{* * * *}$ & $919 / 18^{* * * 4}$ & $|V W V / 4|^{* * *}$ & | | & 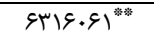 & $19 \cdot / 9 \varphi^{* * * a}$ & $\mid M \Lambda \varepsilon / \varepsilon^{*}{ }^{* * *}$ & 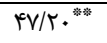 & 1 & سال \\
\hline $1.191^{6}$ & rAT/vq & $r T / Q \varphi$ & $M F / g \Lambda$ & V/VQ & $r \Lambda T / N T$ & FTHETIS. & $|r / V|^{c}$ & $199 / 94$ & T/QT & t & |شتباه 1 \\
\hline$r \mid \varepsilon / \varphi^{* * *}$ & $\Gamma \cdot \kappa T / V \varepsilon^{* * *}$ & $\Delta N / \widetilde{T} V^{* * *}$ & $\Delta \cdot V / T \varphi^{* * * *}$ & & $T V G \cdot / \Delta Q^{* *}$ & $r q \Delta \Delta S \mid N / \mu \cdot{ }^{* * *}$ & 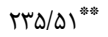 & $\mid f V V / \cdot V^{* * *}$ & $r \Delta \cdot / v \varphi^{* * *}$ & 91 & رُنوتيبِ \\
\hline 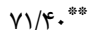 & $\Lambda \cdots / r \cdot{ }^{*}$ & I & س & $109 / 1 .{ }^{*}$ & $|r \varepsilon| / \cdot 1^{* * 3}$ & rAreq /l. & $\mid \varepsilon / \Delta \Lambda$ & $11 \cdot / 99^{* \prime}$ & $\mid V / \Delta T^{* * * *}$ & Ve & رنوتيب × سال \\
\hline r & (т)/^. & $\mid r / g t^{\circ}$ & $s \pi / \pi \Delta$ & re/vq & $r \Delta F / Q Y$ & $\Delta F \mid S A T / Q$. & $|9 / \pi|$ & $I T T / Q T$ & $8 / 4$ & Fre & اشتباه r \\
\hline
\end{tabular}

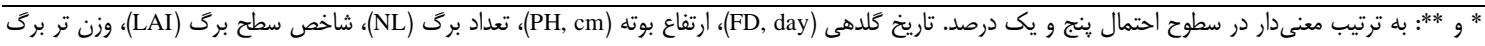

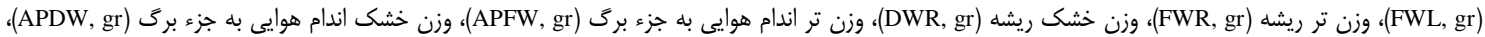
وزن خشك بركَهاى بوته (DLYP, gr).

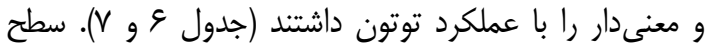

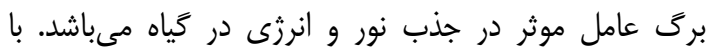

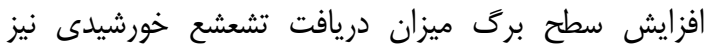

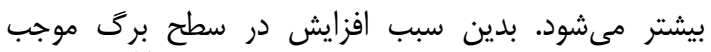

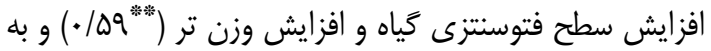

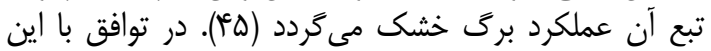

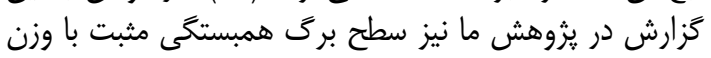

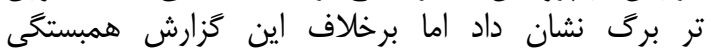

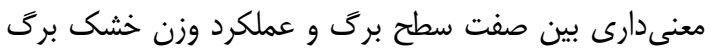

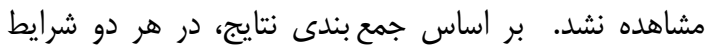

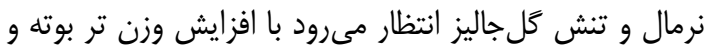

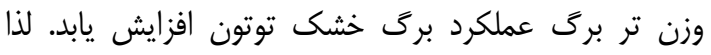

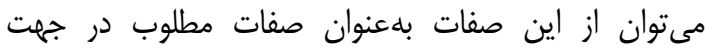

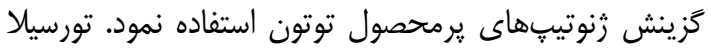

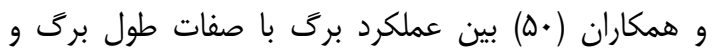

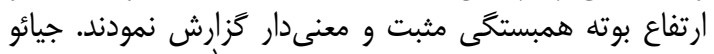

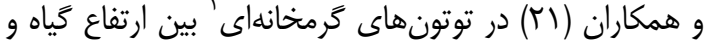

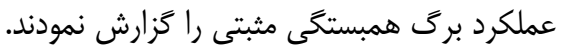

تجزيه همبستكى

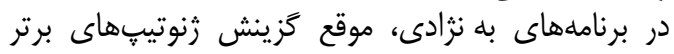

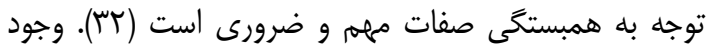

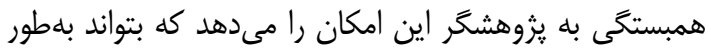

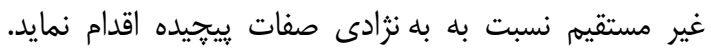

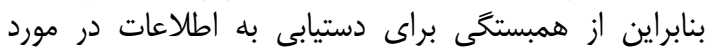

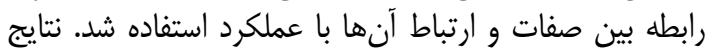

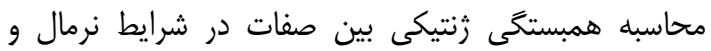

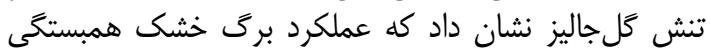

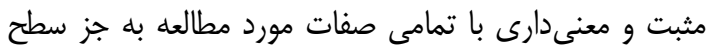

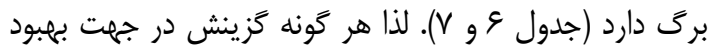

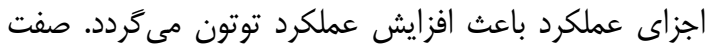

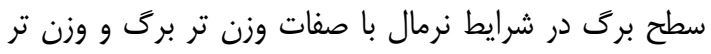

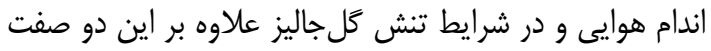

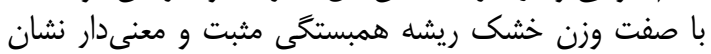

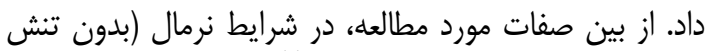

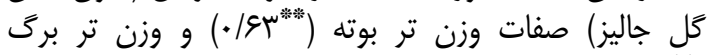
(

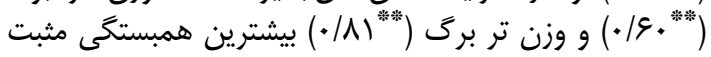

جدول و- همبستكى زنوتيبى بين صفات مورد مطالعه در زنوتيٍهاى توتون شرقى و تنباكو تحت شرايط نرمال (بدون تنش كل جاليز) Table 6. Genotypic correlation among studied traits in oriental and water pipe's tobacco genotypes under normal

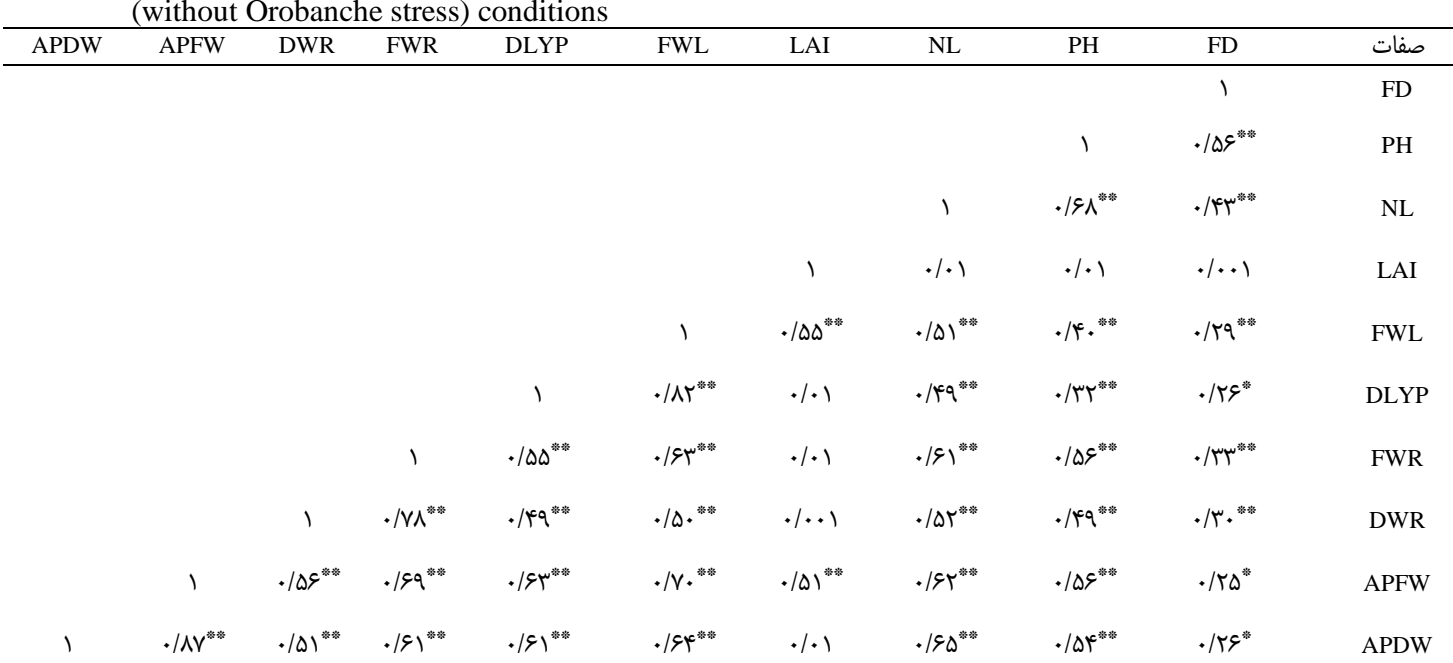

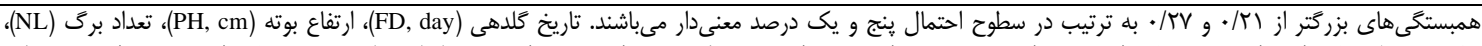

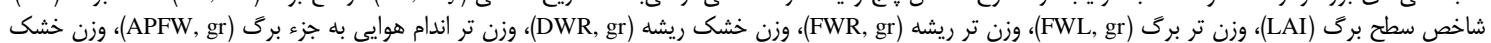

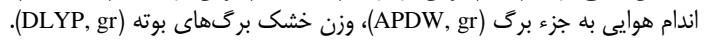


جدول V- همبستخى زنوتيبى بين صفات مورد مطالعه در زنوتيبهاى توتون شرقى و تنباكو تحت تنش گل جاليز Table 7. Genotypic correlation among studied traits in oriental and water pipe's tobacco genotypes under Orobanche

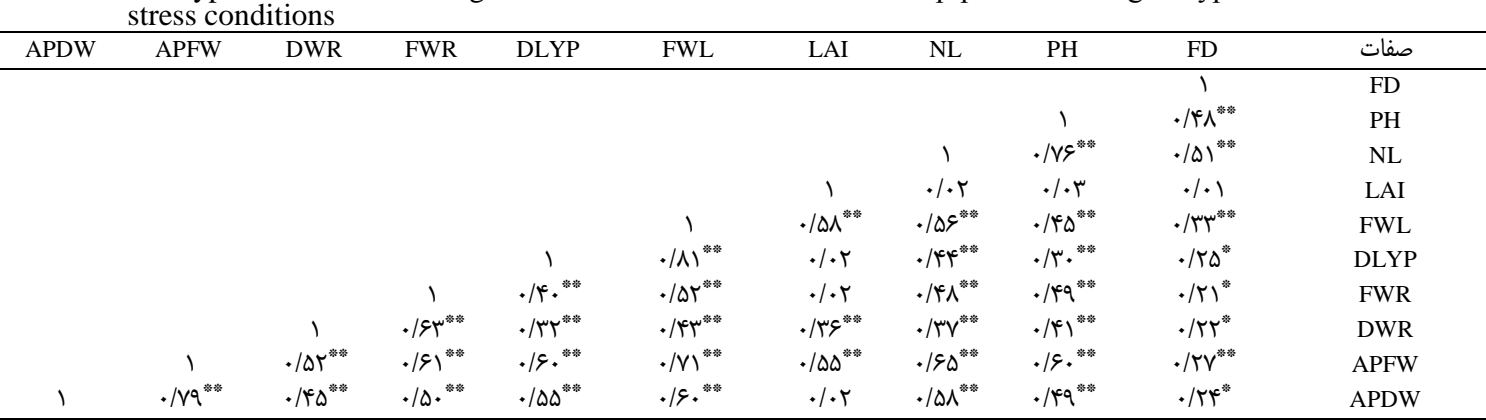

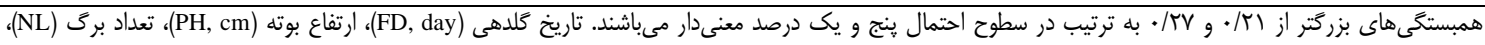

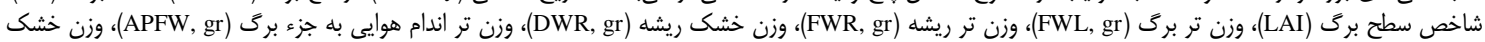

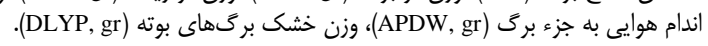

ارزش بيشتر اين خوشه از نظر صفات مهم نظير تعداد برك،

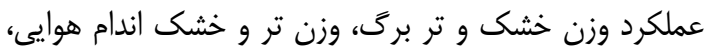

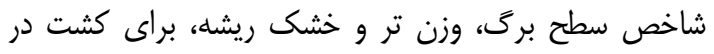

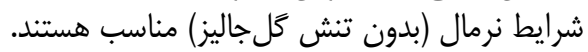

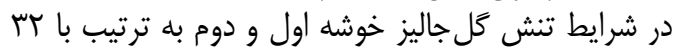

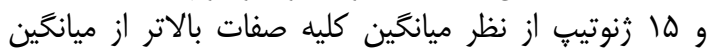

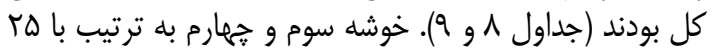

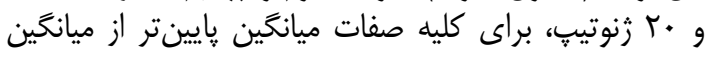
كل داشتند.

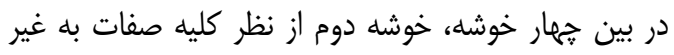

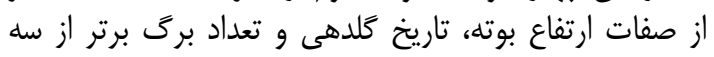

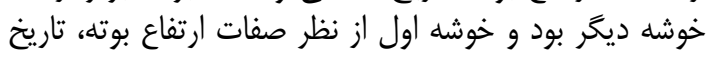

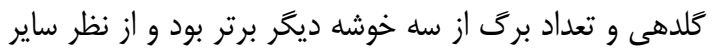

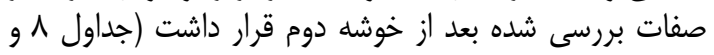

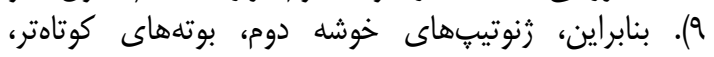

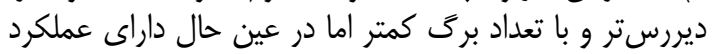

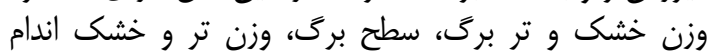

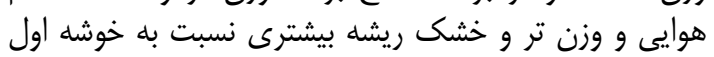

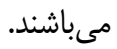

رنوتيبهاى خوشه دوم با توجه به ارزش بيشتر اين خوشه از

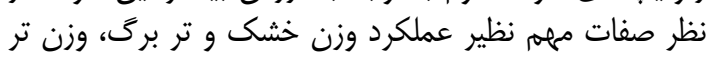

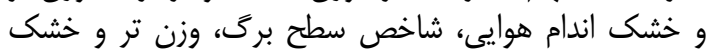

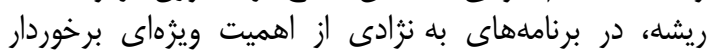

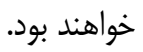

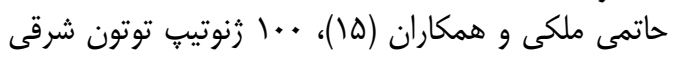

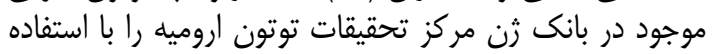

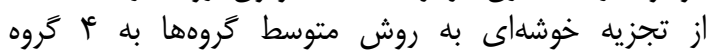

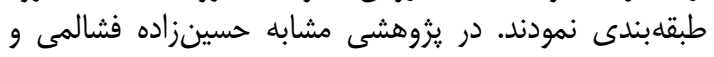

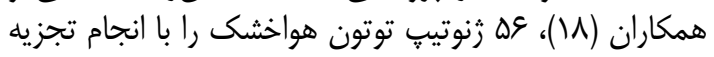

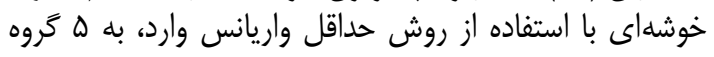

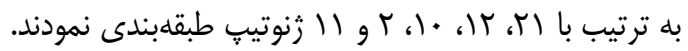

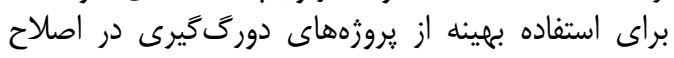

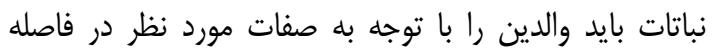

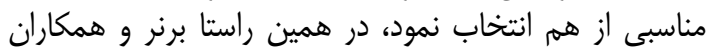

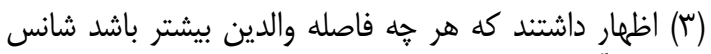
بدست آوردن تنوع افزايش مهيد فاصدا. بررسى تنوع بين
تجزيه خوشهاى ثرنوتيڤهاى توتون شرقى و تنباكو بر اساس صفات مورد

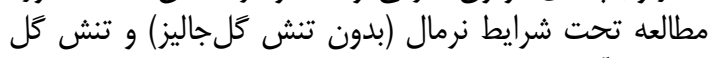

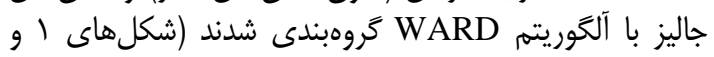

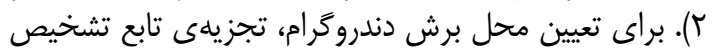

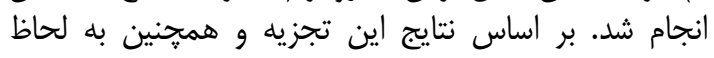

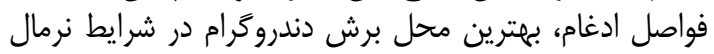

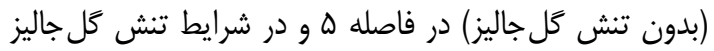

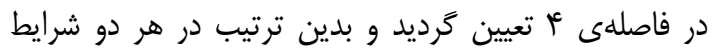

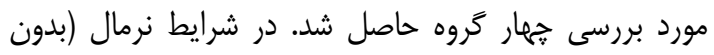

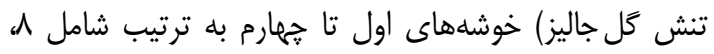

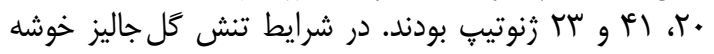

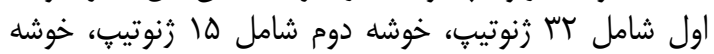

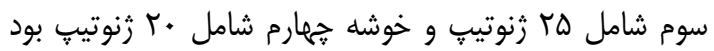

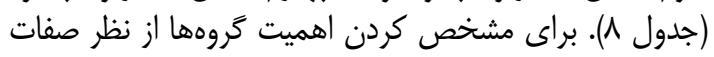

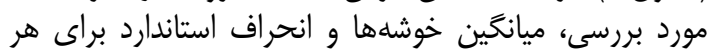

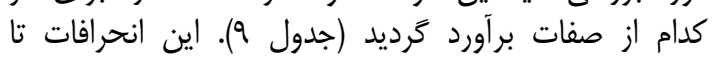

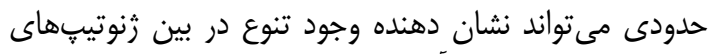

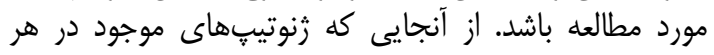

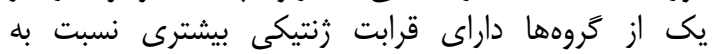

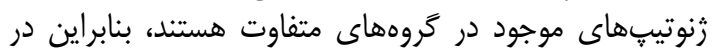

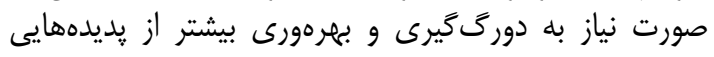

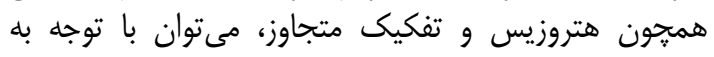

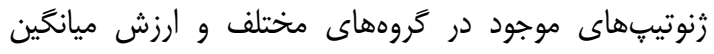
صفات براى هر كروه اقدام به انتخاب نمود درداي

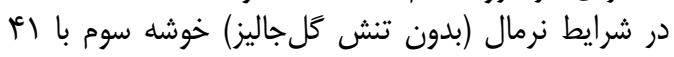

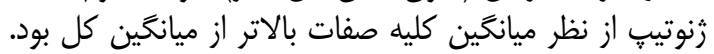

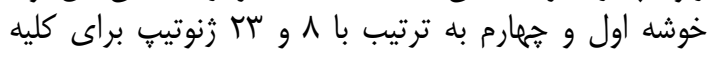

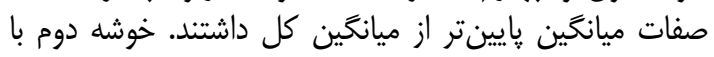

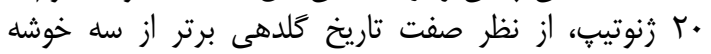

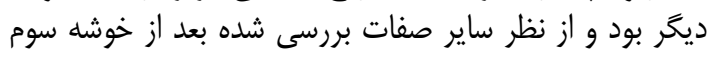

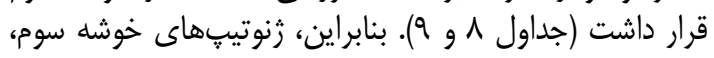

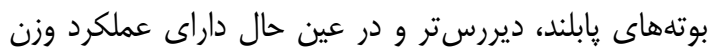

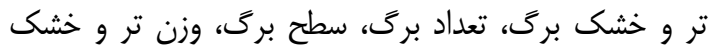

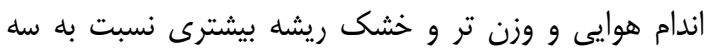

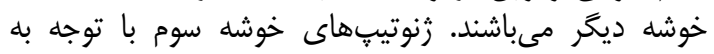




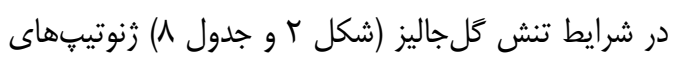

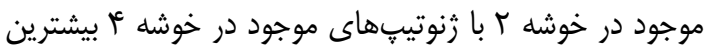

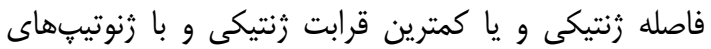

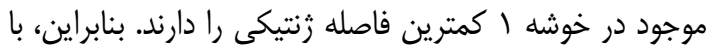

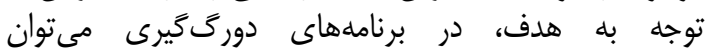

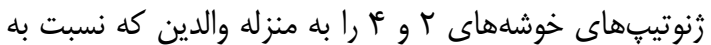

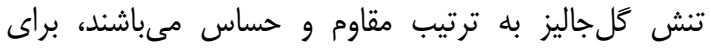

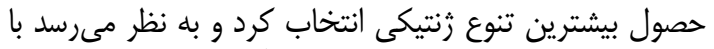

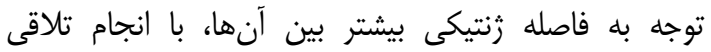

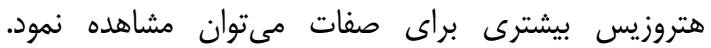

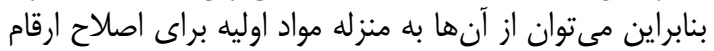

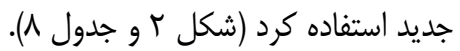

زرنوتيبهاى مختلف بر اساس صفات زراعى اهميت ويزماى تحري

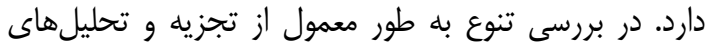

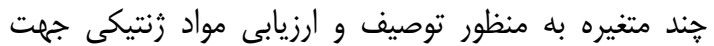
بهره كيرى بهينه و مطالعه روابط داخلى صفات استفاده مى رشود

با توجه نتايج حاصل از تجزيه خوشهاى و رتبايلمابندى

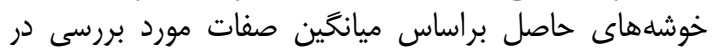

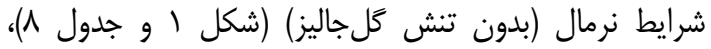

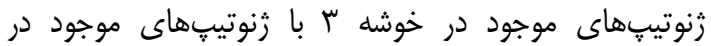

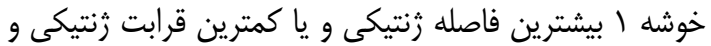

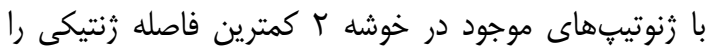
دارند.

جدول ^- رتبه بندى كروههاى حاصل از تجزيه خوشهاى بر اساس ميانكين صفات مورد بررسى در زنوتيبهاى توتون شرقى و تنباكو تحت شرايط نرمال (بدون تنش كل جاليز) و تنش كل جاليز

Table 8. Ranking the groups identified by cluster analysis based on the mean of studied traits in oriental and water pipe's tobacco genotypes under normal (without Orobanche stress) and Orobanche stress conditions

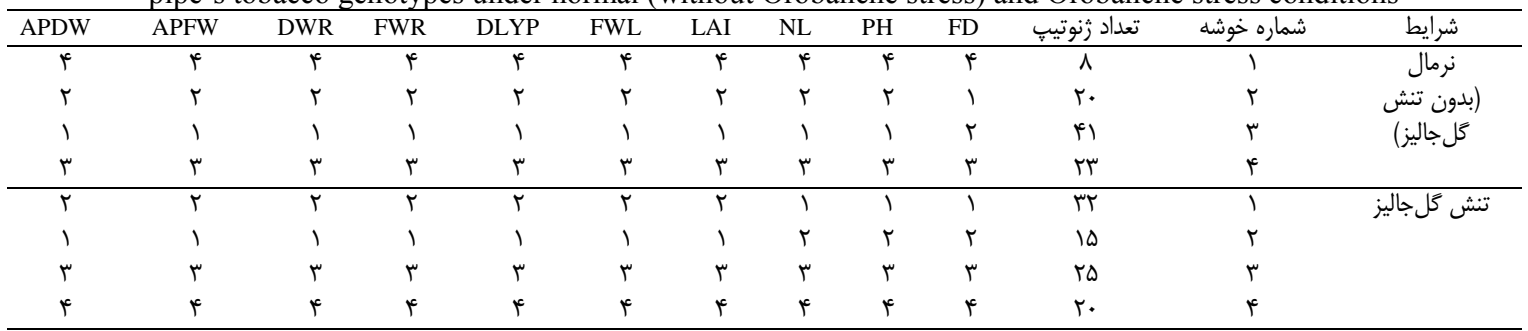

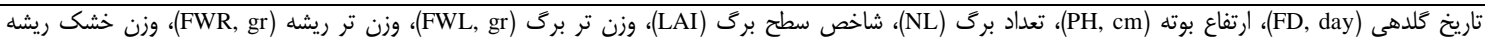

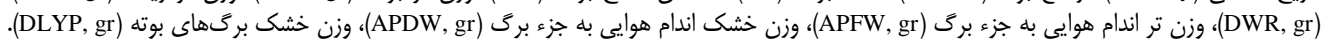

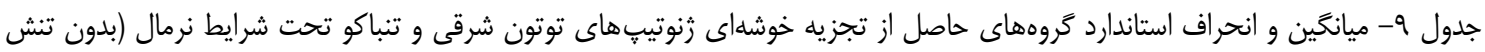

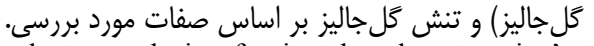

Table 9. Mean and standard deviation of groups resulted from cluster analysis of oriental and water pipe's tobacco genotypes under normal (without Orobanche stress) and Orobanch stress conditions based on the studied traits

\begin{tabular}{|c|c|c|c|c|c|c|c|c|c|c|c|}
\hline APDW & APFW & DWR & FWR & DLYP & FWL & LAI & NL & PH & FD & خوشه & شرايط \\
\hline$r / V I \pm r / 99$ & $\mid r \varepsilon / \Delta \Lambda \pm q / \cdot 1$ & $I V / A V \pm I / \Delta$. & $r N / T \pm r / V T^{c}$ & $r F / r I \pm r / \Delta q$ & $\begin{array}{l} \pm I r / V G \\
I T \Delta /{ }^{\prime} \varepsilon\end{array}$ & $m \| \varepsilon / \Lambda F \pm m F \Delta / r F$ & : $r / q T \pm I / G Y$ & $\Lambda \Gamma / r \Delta \pm \Delta / 1)$ & $98 / 9 \Gamma \pm r / 9 q$ & 1 & \\
\hline $\mid f / \lambda \Lambda \pm \cdot / V F$ & $\Delta r / F \& \pm r / \cdot 1$ & $N / V T \pm \cdot / F V$ & $r V / r F \pm 1 / F \Lambda$ & $q / \Delta \vee \pm . / 98$ & $r \cdot / \Delta \Delta \pm r / r \mid$ & $|f \cdot r / M 1 \pm q V / q|$ & $r / / 99 \pm \cdot / \lambda r$ & $98 / \% 9 \pm \Gamma / 91$ & $\begin{array}{l} \pm 1 / 14 \\
8 x / \cdot 1\end{array}$ & r & 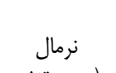 \\
\hline$r \cdot / 4 \wedge \pm \cdot / V I$ & $\Delta N / \cdots \pm \Gamma / ৭ \Delta$ & $|I / r| \pm \cdot / \Delta V$ & $M r / q . \pm 1 / F r$ & $\mid V / r \cdot \pm 1 / \cdot V$ & $9 q / V \cdot \pm f / \cdot 9$ & THYT/KE DITE/OV & $r \Delta / V V^{\top} \pm \cdot / q q$ & $V Y / \Delta S \pm r / A r$ & बr/৭९ $\pm 1 / 1 \Delta$ & r & كُ جاليز) \\
\hline$q / \wedge \varepsilon \pm \cdot / \Delta V$ & $r N / r \backslash \pm T / V T^{c}$ & $\Delta / \Delta) \pm \cdot / \bar{c}$. & $\mid V / \cdot r \pm I / \cdot r$ & $\varepsilon / F \Delta \pm \cdot / \Delta V$ & $r V / Q T \pm Y / \cdot r^{c}$ & $1 \cdots q / r \pi \pm 11 / / \Lambda F$ & $|F / \Delta| \pm \cdot / \Lambda F$ & $r r / q q \pm r / 1$. & $\Delta \Gamma / \wedge \Delta \pm 1 / \Gamma)$ & c & \\
\hline$|V / \&| \pm \cdot / \Lambda T$ & $V \cdot / q T \pm r / 4)$ & $q / \wedge r \pm \cdot / 1 q$ & rq/qr & $\mid f / r \Lambda \pm 1 / .$. & $\Delta V / V \& \pm r / \& \Delta$ & $1119 / 1 \cdot \pm 99 / 4$. & $r Y / \& D \pm \cdot / r)$ & ST/RTII/9D & $8 \cdot / 9 \Lambda \pm \cdot / \wedge \Delta$ & كل ل & \\
\hline $\begin{array}{l} \pm r / F q \\
\mid \omega / \cdot r\end{array}$ & $\begin{array}{l} \pm|r / \Delta| \\
9 \cdot / \Delta \Psi\end{array}$ & $q / \bullet \pm r / V V$ & $\begin{array}{l} \pm q / K \varepsilon \\
r V / q .\end{array}$ & $\begin{array}{l} \pm \Delta / 1 . \\
\mid T / R Q\end{array}$ & $\Delta T / V I \pm I D / r T$ & $\begin{array}{l} \pm r q 4 / 1 q \\
|\varepsilon| q / r r\end{array}$ & $\begin{array}{l} \pm r / q q \\
r \Delta / v q\end{array}$ & $\begin{array}{c} \pm 1 \cdot / V^{\mu} \\
V / / r^{r}\end{array}$ & 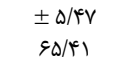 & 1 & \\
\hline $\begin{array}{l} \pm V / G r \\
r \cdot / \Delta .\end{array}$ & $\begin{array}{l} \pm r \Delta / \cdot V \\
V Q / \wedge r\end{array}$ & $\begin{array}{l} \pm r / T \Delta \\
1 . / V^{c}\end{array}$ & $\begin{array}{l} \pm s / r \Lambda \\
r \Psi / \Delta V\end{array}$ & $\begin{array}{l} \pm V / F Y \\
T r / \Delta F\end{array}$ & $V \varepsilon / V \Psi \pm N / \Delta \Delta$ & $\begin{array}{l} \pm \wedge F \cdot / q \Lambda \\
Y \leftarrow \mid V / q \mu\end{array}$ & $\begin{array}{l} \pm T / T \Lambda \\
r \Delta / r \Lambda\end{array}$ & $\Delta V / \Delta \cdot \pm q / \widetilde{\sim} \Delta$ & $\begin{array}{l} \pm F / R \varepsilon \\
g r / M\end{array}$ & r & \\
\hline $\begin{array}{l} \pm r / r \\
1.1 .9\end{array}$ & $\begin{array}{l} \pm \| / 1 / 4 x \\
t \cdot / \mu \Lambda\end{array}$ & $G / Y^{C E} \pm 1 / 8 D$ & $\begin{array}{l} \pm \varepsilon / \wedge q \\
r F / . r\end{array}$ & $q / \mu v \pm r / \Delta \omega^{\circ}$ & & $\begin{array}{l} \pm r \Delta V / \Delta F \\
|\| F q / q|\end{array}$ & $\begin{array}{l} \pm r / \Delta \Lambda \\
19 / r q\end{array}$ & $\begin{array}{l} \pm \mid r / \Delta \Lambda \\
\Delta F / T K\end{array}$ & $\begin{array}{l} \pm s / V I \\
\Delta N / T \Lambda\end{array}$ & r & تنش كَلجاليز \\
\hline G/א & $r \Delta / q q \pm V / \cdot r$ & r/^q $\pm \cdot / 91$ & $\begin{array}{l} \pm r / q r \\
11 / V^{c}\end{array}$ & $\Delta / \digamma^{\epsilon} \cdot \pm 1 / \& \Delta$ & $r \Psi / \Lambda \pm N / \Psi G$ & $V \cdot q / 1 r \pm r \Delta r / \cdot \Lambda$ & $\begin{array}{l} \pm T / F r \\
I T / \Delta D\end{array}$ & $r g / q \vee \pm s / v 1$ & $\begin{array}{l} \pm s / \varepsilon q \\
\Delta s / . r\end{array}$ & te & \\
\hline $\begin{array}{l} \pm s / 19 \\
1 T / V .\end{array}$ & $\begin{array}{c} \pm r r / \cdot V \\
0 \cdot / 9 q\end{array}$ & $V / \Delta \cdot \pm r / \backslash \Lambda$ & $\begin{array}{l} \pm q / F v \\
r F / l q\end{array}$ & $\begin{array}{l} \pm V / I f \\
11 / 99\end{array}$ & $r \Delta / q r \pm r r / \cdot \Delta$ & $\begin{array}{l} \pm V E V / F F \\
\mid F T / G T\end{array}$ & $\begin{array}{l} \pm s / \Delta \mid \\
r \mid / \cdot 9\end{array}$ & $\begin{array}{l} \pm 1 \varepsilon / \Gamma \mu \\
\Delta \xi / q \Gamma\end{array}$ & $\begin{array}{l} \pm \varepsilon / 89 \\
q 1 / \cdot r\end{array}$ & 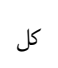 & \\
\hline
\end{tabular}

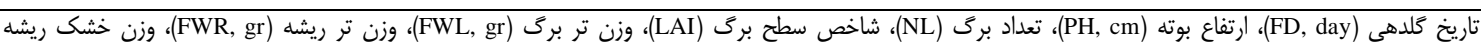

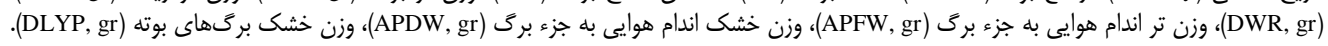




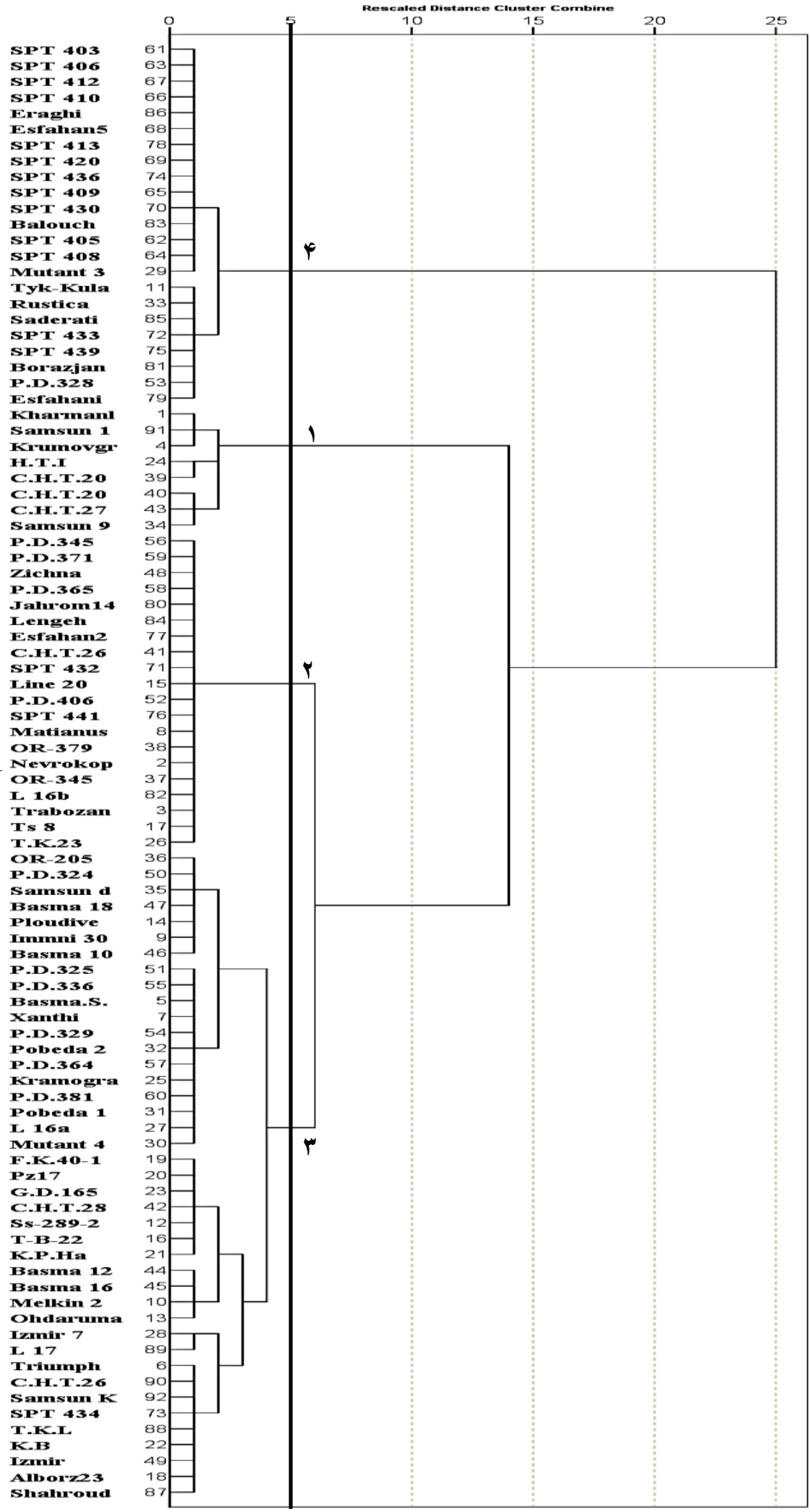

شكل ا - دندوگرام حاصل از گروهبندى زنوتيٍِهاى توتون شرقى و تنباكو تحت شرايط نرمال (بدون تنش گل جاليز) با استفاه از روش وارد Figure 1. Dendrogram of grouping oriental and water pipe's tobacco genotypes under normal (without Orobanche stress) conditions using Ward's method 
SPT 430

SPT 436

Balawel.

Slkabroud

TIXI

SPT 412

$\underset{S P T}{S T 134}$

SPT 403

SPT 441

Fraglai

Rustica

Esfiluain

Esfalkani
SPT 420

SPT 413

SPT 433

SPT 439

SPT 409

Borazjaik

PD 328

Triwmel.

PDA06

Saderati

SPT 406

Mutant 3

PD 345

L 16 :

OR-345

Pasima 181-8

PD320

PD371

Esfalkam2

OR-zOs

SPT 410

SPT 405

Esfalkams

K.E

C.1.T_ $266-6$

Imumui 3000

Izmix: 7

Izmix

PD 365

Tylc-Kun

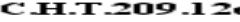

C.Y.T 209.1 2e $\times$ F.K.40

H.T.I

Krumoveraid

T-B-22

Amborz:3

Samsum

KAPAa

GD_165

C.H.T_269-1 20

Samisum katemiz

Melkim 261

F.K.40-1

Basma 1 2-2

Matiamus

Lemselk

Newrolkop

Jahuomi 4

OR-379

Basma.S 31

PD 364

Pobeda 2

PD 381

KXamograd N_H.H. 659

Kovamandi 163

L $16 a$

Pzi 7

T.K.23

Trabozan

PD 336

Xanthi

Basma $16-10$

Ziclema

Oledaxum:

MTutant 4

Pobeda 1

Basma 104-1

L 17

Lime 20

Sams 959

Ts 8

Plomdive 58

PD 324

Samisul dere

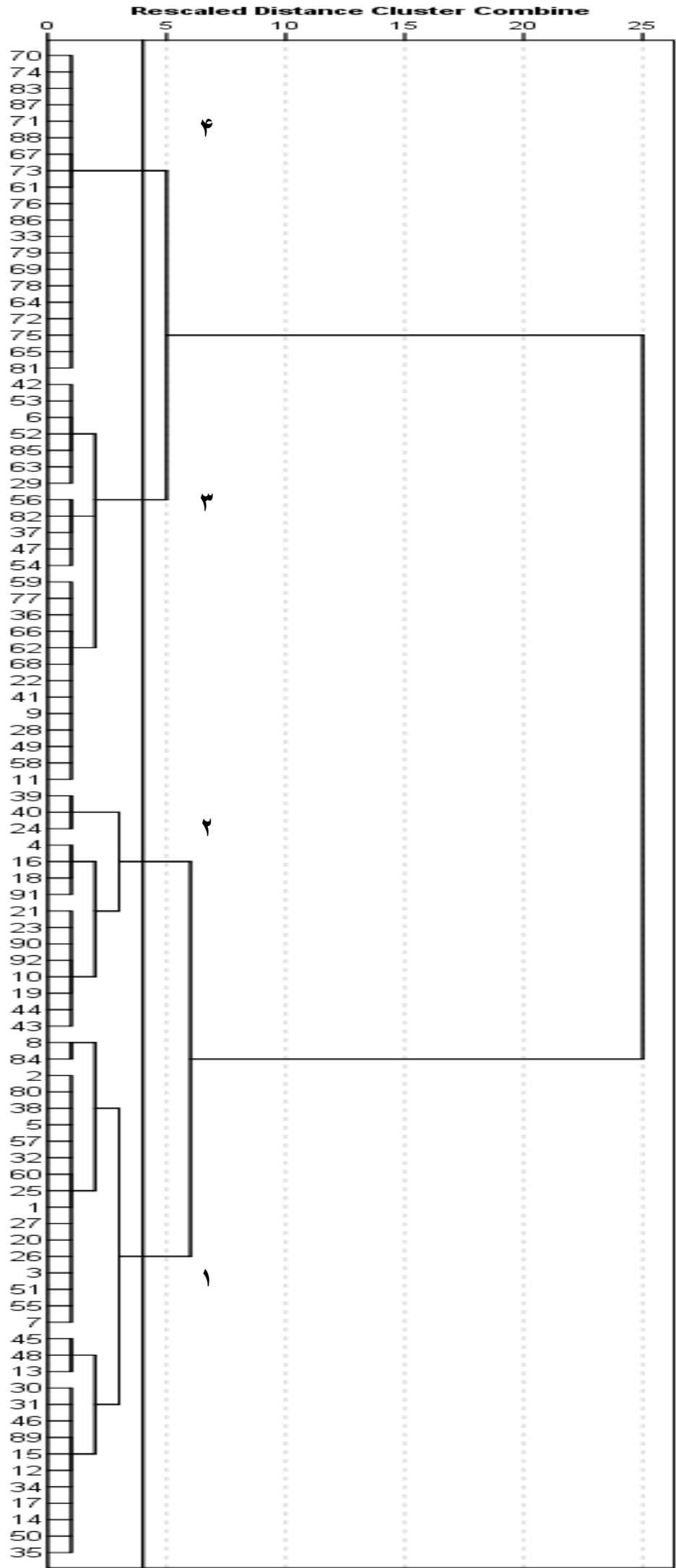

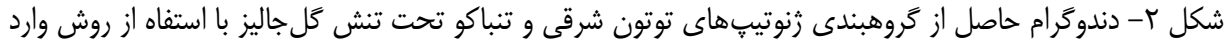

Figure 2. Dendrogram of grouping oriental and water pipe's tobacco genotypes under Orobanche stress conditions using Ward's method 


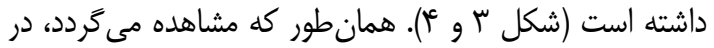

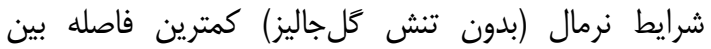

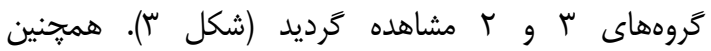

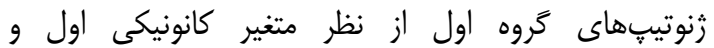

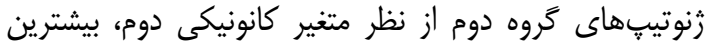

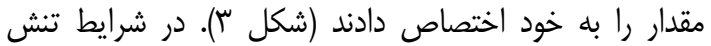

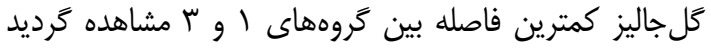

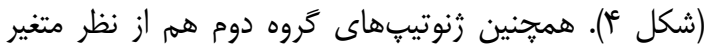

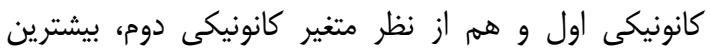
مقدار را به خود اختصاص دادند (شكل أ).

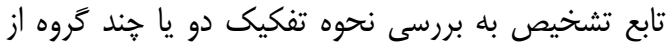

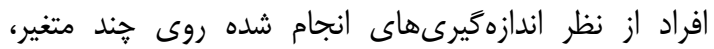

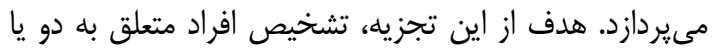

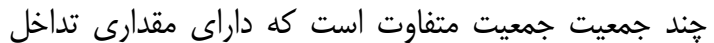

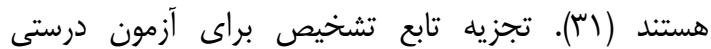

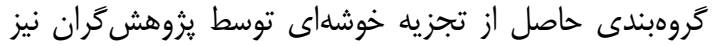

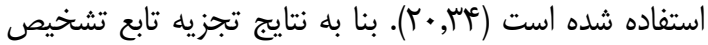

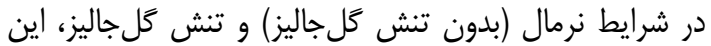

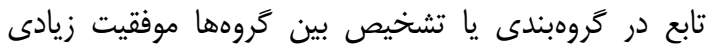

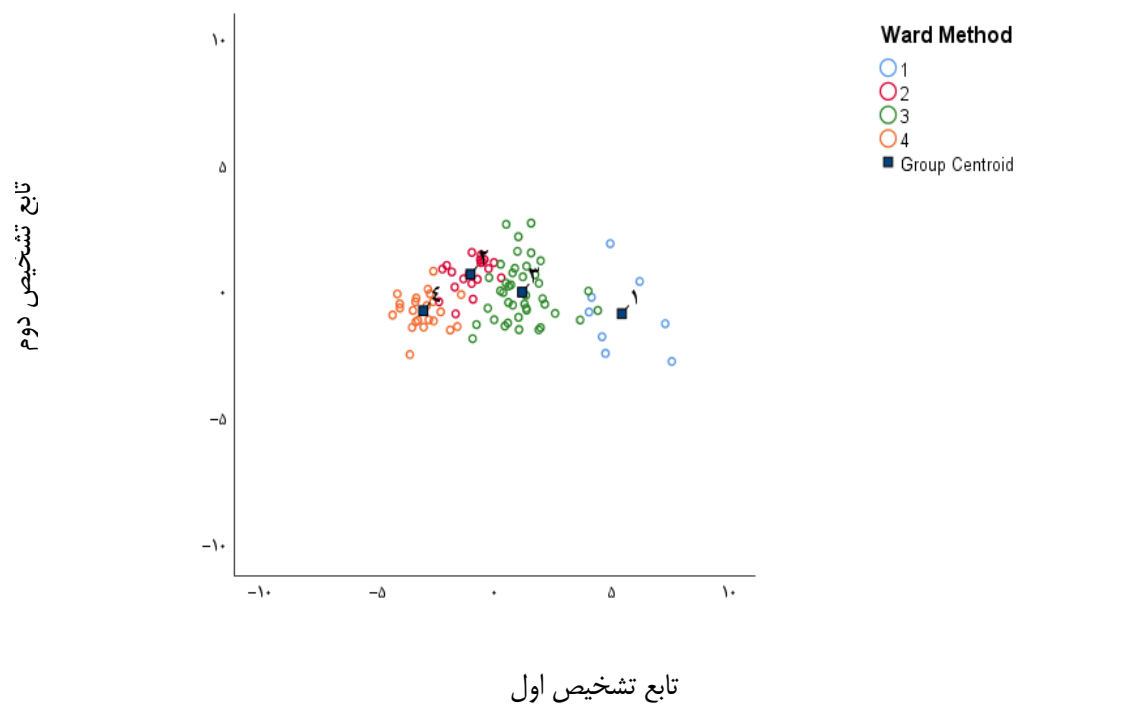

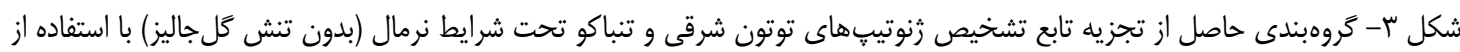

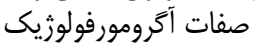

Figure 3. The results from discriminant function analysis for oriental and water pipe's tobacco genotypes under normal (without Orobanch stress) condition using agro-morphological traits

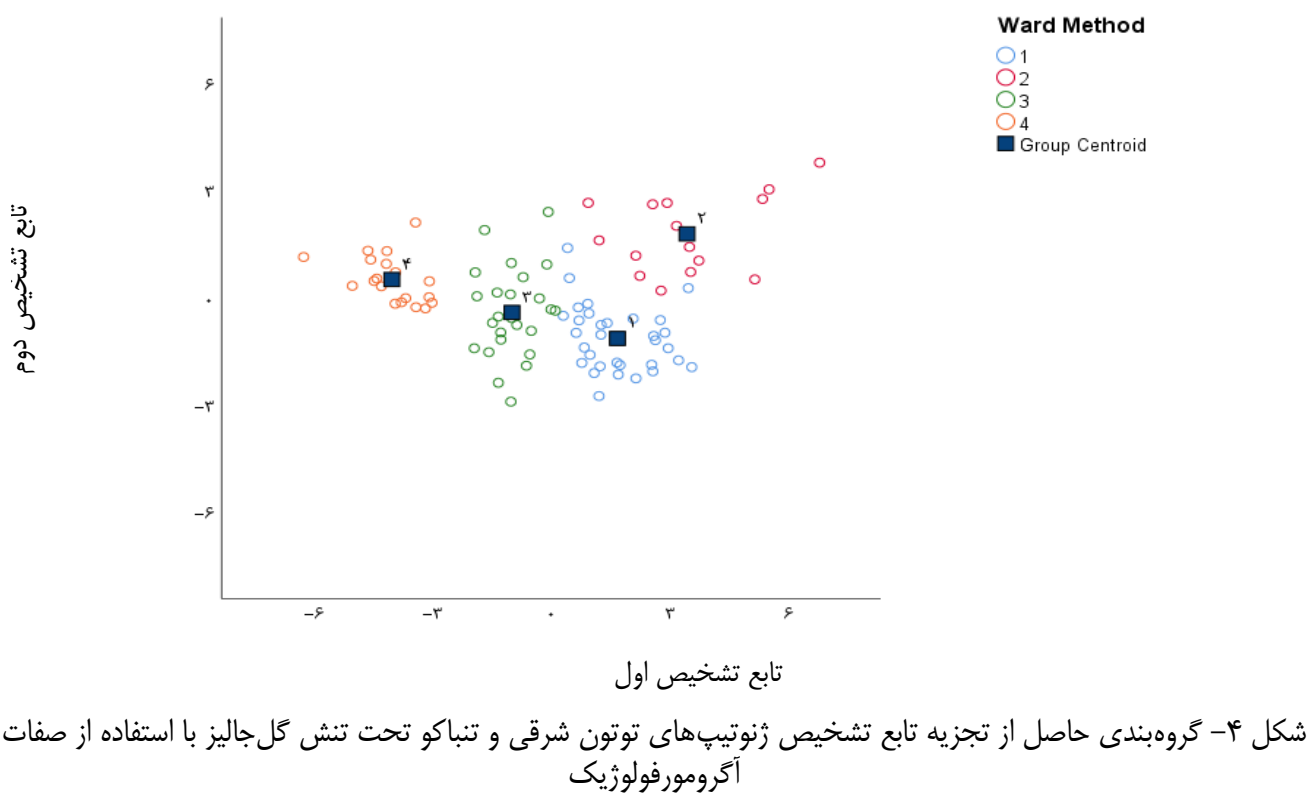

Figure 4. The results from discriminant function analysis for oriental and water pipe's tobacco genotypes under Orobanche stress conditions using agro-morphological traits 
مربوط به ويزگگهاى وزن تر و خشك برك بود. بنابراين

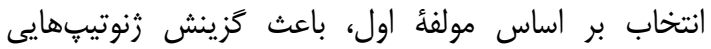

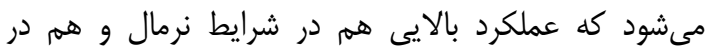

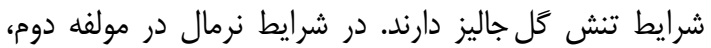

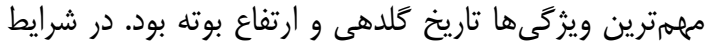

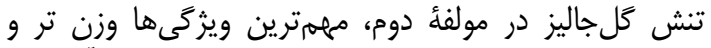

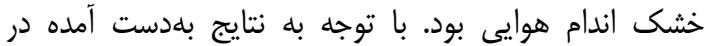

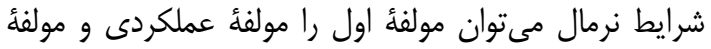

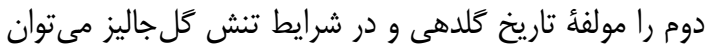

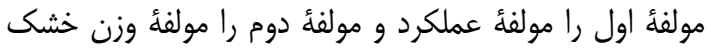
اندام هوايى تعريف نمود. سودمندى استفاده از مولئ مولفهئهاى

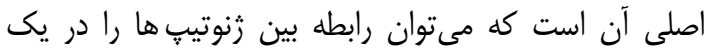

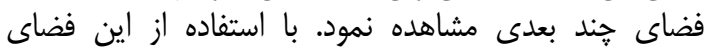

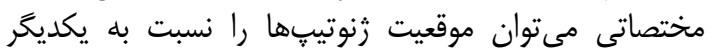
شناسايى نمود.
تجزيه بله مولفههاى اصلى تجلى

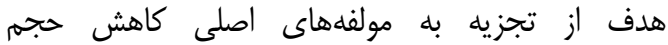

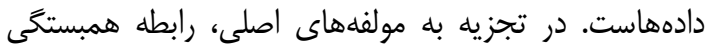

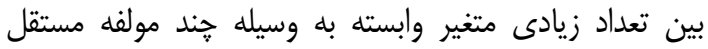

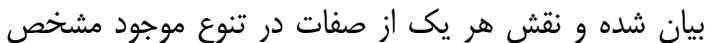

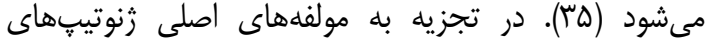

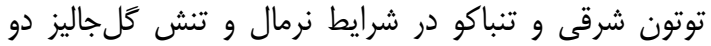

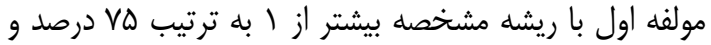

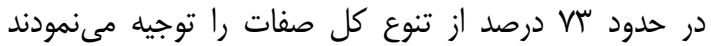

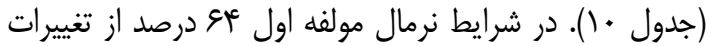

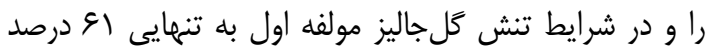

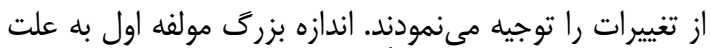

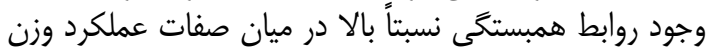

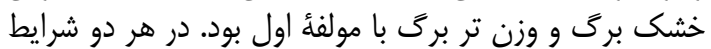

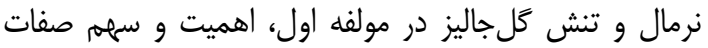
در توجيه تنوع زنتيكى كه بزركترين ضرايب رالين را دارا بودند

جدول • ا- ضرايب تجزيه به مولفههاى اصلى براى صفات مورد بررسى در زنوتيبهاى توتون شرقى و تنباكو تحت شرايط نرمال (بدون تنش كل جاليز) و تنش كَل جاليز

Table 10. Principal component coefficients of studied traits in oriental and water pipe's tobacco genotypes under normal (without Orobanche stress) and Orobanch stress conditions.

\begin{tabular}{|c|c|c|c|c|c|c|c|c|c|c|c|c|}
\hline \multirow{2}{*}{ واريانس تجمعى (\%) } & \multicolumn{10}{|c|}{ ضرايب مربوط به صفات موجود در داخل هر مولفه } & \multirow{2}{*}{ 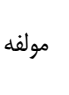 } & \multirow{2}{*}{ 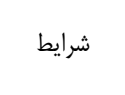 } \\
\hline & APDW & APFW & DWR & FWR & DLYP & FWL & LAI & NL & PH & FD & & \\
\hline$\cdot / a t$ &.$/ \mathrm{V}$ & $\cdot / \mathrm{A}$ & .1 .9 &.$/ 11$ & $\cdot / T \Lambda$ & . & $\cdot|r|$ & $\cdot /$. & $-\cdot / / V$ &.$- / 4 T$ & 1 & 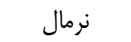 \\
\hline$\cdot / V \Delta$ &.$- / . r$ &.$- / \cdot r$ & $\cdot$. & $\cdot 1 \cdot 1$ & $-\cdot / 4$ &.$- / / V$ &.$- / 11$ & $\cdot / K F$ & $\cdot / 4 q$ & $\cdot|\Delta|$ & r & \\
\hline.$|9|$ & . & $\cdot / \Gamma \Delta$ & $\cdot / 1 \Lambda$ & 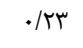 &.$/ 91$ & .199 & $\cdot / \mu r$ & . & .1 .9 & $\cdot / 4$ & 1 & تنش كل جاليز \\
\hline.$/ N^{\mu}$ & $\cdot / \Lambda \varepsilon$ &.$/ v$ & .119 & r &.$/ T V$ & وس/. &.$/ \Gamma \Lambda$ & . & . & .111 & r & \\
\hline
\end{tabular}

از بين صفات مورد مطالعه، در شرايط نرمال (بدون گل جاليز)

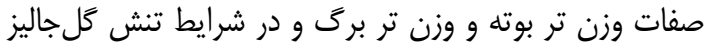

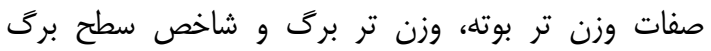

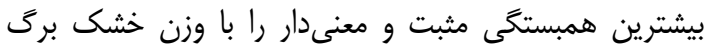

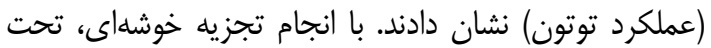

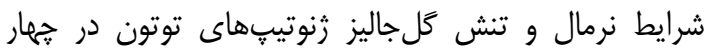

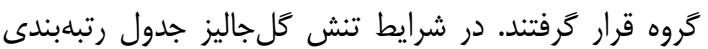

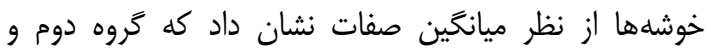

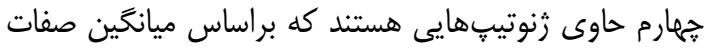

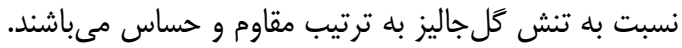

\section{جمعبندى نتايج}

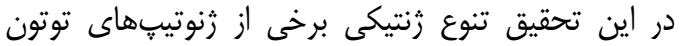

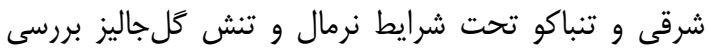

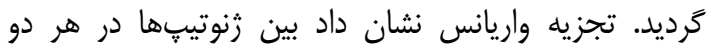

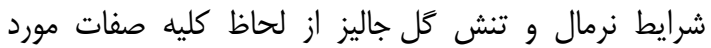

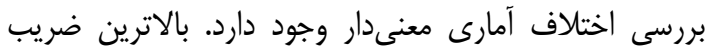

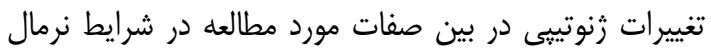

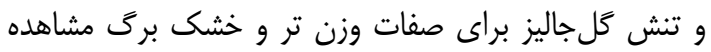

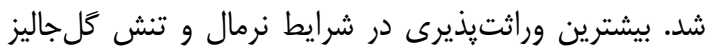

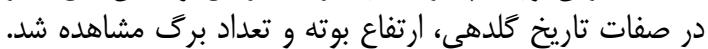

منابع

1. Beikzadeh, H., S.S. Alavi, M. Bayat and A. Ezady. 2015. Estimation of genetic parameters of effective agronomical traits on yield in some of Iranian rice cultivar. Agronomy Journal (Pajouhesh \& Sazandegi), (104): 73-78 (In Persian).

2. Brandle, J. and D. Bai. 1999. Biotechnology: uses and applications in tobacco improvement. In Tobacco: Production, Chemistry Technology. Wiley-Blackwell: Oxford, UK. 49-65.

3. Brenner, D., D. Baltensperger, P. Kulakow, J. Lehmann, R. Myers, M. Slabbert and B. Sleugh. 2000. Genetic resources and breeding of Amaranthus. Plant Breeding Reviews, 19: 227-285.

4. Falconer, D.S. and T.F.C. Mackay. 1996. Introduction to Quantitative Genetics. 4th Edition, Addison Wesley Longman, Harlow. 
5. Davalieva, K., I. Maleva, K. Filiposki, O. Spiroski and G.D.J.D. Efremov. 2010. Genetic variability of Macedonian tobacco varieties determined by microsatellite marker analysis. Diversity, 2(4): 439-449.

6. Echevarría-Zomeño, S., A. Pérez-de-Luque, J. Jorrín and A.M. Maldonado. 2006. Pre-haustorial resistance to broomrape (Orobanche cumana) in sunflower (Helianthus annuus): cytochemical studies. Journal of Experimental Botany, 57(15): 4189-4200.

7. Eid, M.H.J.I.J.o.G. 2009. Estimation of heritability and genetic advance of yield traits in wheat (Triticum aestivum L.) under drought condition. International Journal of Genetics and Molecular Biology, 1(7): 115-120.

8. Eizenberg, H., J.B. Colquhoun and C.A. Mallory-Smith. 2003. Variation in clover response to small broomrape (Orobanche minor). Weed Science, 51(5): 759-763.

9. FAO. 2017. FAOSTAT. Projections of tobacco production, consumption and trade to the year. Online available at http://faostat.fao.org/site/567/DesktopDefault. aspx? PageID=567\#ancor.

10. Frankham, R., J.D. Ballou and D.A. Briscoe. 2004. A primer of conservation genetics. Cambridge University Press.

11. Giannelos, P., F. Zannikos, S. Stournas, E. Lois and G. Anastopoulos. 2002. Tobacco seed oil as an alternative diesel fuel: physical and chemical properties. Industrial Crops and Products, 16(1): 1-9.

12. Goldwasser, Y., H. Eizenberg, J. Hershenhorn, D. Plakhine, T. Blumenfeld, H. Buxbaum, S. Golan, and Y. Kleifeld. 2001. Control of Orobanche aegyptiaca and O. ramosa in potato. Crop Protection, 20(5): 403-410.

13. Goldwasser, Y. and Y. Kleifeld. 2004. Recent approaches to Orobanche management. Weed Biology and Management. Springer, pp: 439-466.

14. Habimana, S., K. Murthya, V. Hattia and A. Nduwumuremyib. 2013. Management of Orobanche in field crops-a review. Crop Science, 2(11): 144-158.

15. Hatami, M.H., G. Karimzadeh, R. Darvishzadeh and R. Alavi. 2012. Genetic variation of oriental tobaccos using multivariate analysis. Iranian Journal of Field Crops Research, 10(1): 100-106 (In Persian).

16. Holland, J., W. Nyquist and C. Cervantes. 2003. Estimating and interpreting heritability for plant breeding: An update. Plant Breeding Reviews Vol. 22.

17. Holland, J.B. 2006. Estimating genotypic correlations and their standard errors using multivariate restricted maximum likelihood estimation with SAS Proc MIXED. Crop Science, 46(2): 642-654.

18. Hosseinzadeh Fashalami, N., A.R. Mahdavi, N. Moarrefzadeh, S.A. Sajadi and R. Alinejad. 2008. Investigation of genetic diversity and classification of different air-cured tobacco varieties. Research Research Workbook of Tirtash Research and Education Center, 105-126 (In Persian).

19. Hosseinzadeh, F.N., M.Z. Shahadati, G. Kiani, M.R. Salavati, P. Zamani, A. Mahdavi and R. Alinejad. 2015. Investigation of genetic diversity among different oriental tobacco (Nicotiana tabacum L.) varieties using multivariate methods. Journal of Crop Breeding, 7(15): 126-134. (In Persian).

20. Jaynes, D., T. Kaspar, T. Colvin and D. James. 2003. Cluster analysis of spatiotemporal corn yield patterns in an Iowa field. Agronomy Journal, 95(3): 574-586.

21. Jiao, F.C., B.G. Xiao, H.Q. Yu, Y.H. ZHANG and X.P.J.J.H.A.U. LU. 2007. Gray correlation analysis on the main agronomic characters and yield of the flue-cured tobacco. Journal of Gansu Agricultural University, 33(5): 564.

22. Joel, D.M. 2000. The long-term approach to parasitic weeds control: manipulation of specific developmental mechanisms of the parasite. Crop Protection, 19(8-10): 753-758.

23. Johnson, D.E. 1998. Applied multivariate methods for data analysts. Duxbury press Pacific Grove, California.

24. Johnson, H.W., H. Robinson and R. Comstock. 1955. Estimates of genetic and environmental variability in soybeans. Agronomy Journal, 47(7): 314-318.

25. Khalili, M.N. and Mohammad Reza 2018. Evaluation of Genetic Diversity of Spring Wheat Cultivars for Physiological and Agronomic Traits under Drought Stress. Journal of Crop Breeding, 10(25): 138151 (In Persian).

26. Labrousse, P., M. Arnaud, H. Serieys, A. Bervillé and P. Thalouarn. 2001. Several mechanisms are involved in resistance of Helianthus to Orobanche cumana Wallr. Annals of Botany, 88(5): 859-868.

27. Laurentin, H. 2009. Data analysis for molecular characterization of plant genetic resources. Genetic Resources and Crop Evolution, 56(2): 277-292.

28. Lowe, A., S. Harris and P. Ashton. 2009. Ecological genetics: design, analysis, and application. John Wiley \& Sons.

29. Lozano, M., M. Moreno, D. Rubiales and A. Perez-De-Luque. 2007. Medicago truncatula as a model for non-host resistance in legume-parasitic plants interactions. Plant Physiology, 145: 437-449.

30. Mariam, E.G. and R. Suwanketnikom. 2004. Screening of tomato (Lycopersicon esculentum Mill.) varieties for resistance to branched broomrape (Orobanche ramosa L.). Kasetsart Journal, 38: 434439.

31. Moghaddam, M., A. Mohammadi-Shoti and M. Aghaei-Sarbarzeh. 1994. Introduction to multivariate statistical methods. J Sci. Vanguard Publishers, Tabriz. Iran (In Persian). 
32. Mohammadi, S., B. Prasanna and N. Singh. 2003. Sequential path model for determining interrelationships among grain yield and related characters in maize. Crop Science, 43(5): 1690-1697.

33. Mohammadi, S.A. and B. Prasanna. 2003. Analysis of genetic diversity in crop plants-salient statistical tools and considerations. Crop Science, 43(4): 1235-1248.

34. Moreda-Pineiro, A., A. Fisher and S.J. Hill. 2003. The classification of tea according to region of origin using pattern recognition techniques and trace metal data. Journal of Food Composition and Analysis, 16(2): 195-211.

35. Pearson, K. 1901. LIII. On lines and planes of closest fit to systems of points in space. The London, Edinburgh, and Dublin Philosophical Magazine and Journal of Science, 2(11): 559-572.

36. Pérez-de-Luque, A., C.I. González-Verdejo, M.D. Lozano, M.A. Dita, J.I. Cubero, P. GonzálezMelendi, M.C. Risueño and D. Rubiales. 2006. Protein cross-linking, peroxidase and $\beta-1$, 3endoglucanase involved in resistance of pea against Orobanche crenata. Journal of Experimental Botany, 57(6): 1461-1469.

37. Pérez-de-Luque, A., M.D. Lozano, J.I. Cubero, P. González-Melendi, M.C. Risueno and D. Rubiales. 2006. Mucilage production during the incompatible interaction between Orobanche crenata and Vicia sativa. Journal of Experimental Botany, 57(4): 931-942.

38. Perez-De-Luque, A., D. Rubiales, J.I. Cubero, M. Press, J. Scholes, K. Yoneyama, Y. Takeuchi, D. Plakhine and D. Joel. 2005. Interaction between Orobanche crenata and its host legumes: unsuccessful haustorial penetration and necrosis of the developing parasite. Annals of Botany, 95(6): 935-942.

39. Pérez-de-Luque, A., M.D. Lozano, M. Moreno, P. Testillano and D. Rubiales. 2007. Resistance to broomrape (Orobanche crenata) in faba bean (Vicia faba): cell wall changes associated with prehaustorial defensive mechanisms. Annals of Applied Biology, 151(1): 89-98.

40. Perez de Luque, A., J. Jorrin, J. Sillero, J. Cubero, D. Rubiales, A. Fer, P. Thalouran, D. Joel, L. Musselman and C. Parker. 2001. Differences in resistance to Orobanche crenata in Pisum spp. Description at the different developmental stages of the parasite and correlationship with host peroxidase. In: Proceedings 7th International Parasitic Weed Symposium. Nantes, 235 pp.

41. Petrova Bozhinova, R. 2006. Coefficients for determination of the leaf area in three Burley tobacco varieties. Journal of Central European Agriculture, 7(1): 7-12.

42. Rispail, N., M.A. Dita, C. González-Verdejo, A. Pérez-de-Luque, M.A. Castillejo, E. Prats, B. Román, J. Jorrín and D. Rubiales. 2007. Plant resistance to parasitic plants: molecular approaches to an old foe. New Phytologist, 173(4): 703-712.

43. Román, B., R. Hernández, A.J. Pujadas-Salvá, J.I. Cubero, D. Rubiales and Z. Satovic. 2007. Genetic diversity in two variants of Orobanche gracilis Sm.[var. gracilis and var. deludens (Beck) A. Pujadas](Orobanchaceae) from different regions of Spain. Electronic Journal of Biotechnology, 10(2): 221-229.

44. Rubiales, D., C. Alcántara and J. Sillero. 2004. Variation in resistance to Orobanche crenata in species of Cicer. Weed Research, 44(1): 27-32.

45. Salavati, M.R., H. Abbasi, N. Hossinzadeh and R. Alinejad. 2005. Depreciatory ability production seedling lateral of flue-cured Tobacco using linebreeding. Research Workbook of Tirtash Research and Education Center, 105-110 (In Persian).

46. Serghini, K., A.P. de Luque, M. Castejón-Muñoz, L. García-Torres and J.V. Jorrín. 2001. Sunflower (Helianthus annuus L.) response to broomrape (Orobanche cernua Loefl.) parasitism: induced synthesis and excretion of 7-hydroxylated simple coumarins. Journal of Experimental Botany 52(364): 2227-2234.

47. Sillero, J., M. Moreno and D. Rubiales. 2005. Sources of resistance to crenate broomrape among species of Vicia. Plant Disease, 89(1): 23-27.

48. Singh, R. and B. Chaudhary. 1985. Biometrical Methods in Quantitative Genetic Analysis. Ludhiana. New Delhi: Kalyani Publishers.

49. Stansfield, W.D. 1991. Theory and Problems in Genetics. McGraw-Hill.

50. Torrecilla, G.G., C.L.B. Del and L.A. Pino. 2002. Correlation in guantitative variable ofblack type tobacco. Cuba Tobacco, 32: 29-36.

51. Troyer, A., S. Openshaw and K. Knittle. 1988. Measurement of genetic diversity among popular commercial corn hybrids. Crop Science, 28(3): 481-485. 


\title{
Study on Genetic Diversity of Some Oriental and Water Pipe's Tobacco Genotypes (Nicotiana Tabacum L.) Under Orobanche Stress Conditions by Using Multivariate Statistical Methods
}

\author{
Maryam Tahmasbali ${ }^{1}$, Amir Fayaz Moghaddam ${ }^{2}$, Reza Darvishzadeh $^{3}$ and \\ Hossein Abbasi Holasou ${ }^{4}$
}

\author{
1- PhD Student in Plant Breeding, Department of Plant Production and Genetics, Faculty of Agriculture and Natural \\ Resources, Urmia University, Urmia, Iran \\ 2- Associate Professor, Department of Plant Production and Genetics, Faculty of Agriculture and Natural Resources, \\ Urmia University, Urmia, Iran, (Corresponding author: a.fmoghaddam@urmia.ac.ir) \\ 3- Professor, Department of Plant Production and Genetics, Faculty of Agriculture and Natural Resources, Urmia \\ University, Urmia, Iran \\ 4- Graduted PhD Student in Plant Breeding, Department of Plant Breeding and Biotechnology, Faculty of \\ Agriculture, University of Tabriz, Tabriz, Iran \\ Received: December 27, 2019 \\ Accepted: April 6, 2020
}

\begin{abstract}
In this research, the genetic diversity of some oriental and water pipe's tobacco genotypes was investigated under normal (without Orobanche) and Orobanche stress conditions during 2007-2008 and 2008-2009 at Urmia Tobacco Research Centre with randomized complete block design with three replications. Combined analysis of variance revealed extent genetic variability among the genotypes for most of the studied traits. This suggests that there is high genetic variability among tobacco genotypes for resistance to Orobanche which can be effectively used in biotic resistance breeding programs. A high genotypic coefficient of variation was seen for studied traits especially for FWL and DLYP in normal and Orobanche stress conditions. The highest broad-sense heritability was estimated for FD, NL and PH in both normal and Orobanche stress conditions. The results of the genetic advance calculation showed that the highest genetic advance was related to LAI, APFW, PH and FWL traits. Based on the results of genetic correlation coefficients of traits, positive and significant genetic correlations were observed between DLYP with all studied traits in both normal and Orobanche stress conditions. Cluster analysis classified all tobacco genotypes into four clusters under normal and stress conditions. In Orobanche stress condition, ratings the clusters in view of traits average showed that the second and fourth clusters contain genotypes that are resistant and sensitive to Orobanche stress, respectively. Therefore, by selecting suitable parents from these groups and crossing them, it will be possible to produce segregated populations in Orobanche resistance breeding programs and to make desirable changes in tobacco yield. Results of cluster analysis were confirmed by results of canonical discriminant function results. The principal component analysis identified two components in each condition which explained more than 75 and 73 percent of the total variation in normal and stress conditions, respectively. Genotypes classified in the second cluster can be potentially used in the Orobanche infested areas.
\end{abstract}

Keywords: Biotic Stress, Breeding For Resistance, Heritability, Multivariate Analysis, Obligate Parasite, Tobacco 